Revue des patrimoines

\title{
L'industrie à domicile depuis l'espace public : essai de typo-chronologie en Montagne thiernoise
}

Brigitte Ceroni et Bénédicte Renaud

\section{(2) OpenEdition}

Journals

Édition électronique

URL : http://journals.openedition.org/insitu/3921

DOI : 10.4000/insitu.3921

ISSN : 1630-7305

Éditeur

Ministère de la culture

Référence électronique

Brigitte Ceroni et Bénédicte Renaud, «L'industrie à domicile depuis l'espace public : essai de typo-

chronologie en Montagne thiernoise », In Situ [En ligne], 9 | 2008, mis en ligne le 18 avril 2012, consulté le 30 avril 2019. URL : http://journals.openedition.org/insitu/3921 ; DOI : 10.4000/insitu.3921

Ce document a été généré automatiquement le 30 avril 2019.

\section{c)}

In Situ Revues des patrimoines est mis à disposition selon les termes de la licence Creative Commons Attribution - Pas d'Utilisation Commerciale - Pas de Modification 4.0 International. 


\title{
L'industrie à domicile depuis l'espace public : essai de typo- chronologie en Montagne thiernoise
}

\author{
Brigitte Ceroni et Bénédicte Renaud
}

\section{Un prolongement nécessaire}

1 A l'issue d'un inventaire du patrimoine industriel de la commune de Thiers, il apparaissait nécessaire de compléter les données en élargissant l'aire d'étude à l'ensemble du bassin industriel ${ }^{1}$. Les recherches avaient montré que la parcellisation du travail de coutellerie, en vigueur surtout au XIX ${ }^{e}$ siècle, avait donné naissance à une spécialisation technique : certaines activités (fabrication des mèches de tire-bouchons, fabrication des manches,...) ne sont pas représentées sur la commune de Thiers, ce qui nuisait à la compréhension du processus de fabrication. La communauté de communes de la Montagne thiernoise, à laquelle a été jointe la commune de Saint-Rémy-sur-Durolle, a constitué ainsi la nouvelle aire d'étude du patrimoine coutelier de la région ${ }^{2}\left(\right.$ fig. $\left.^{\circ}{ }^{\circ} \mathbf{1}\right)$. 
Figure 1

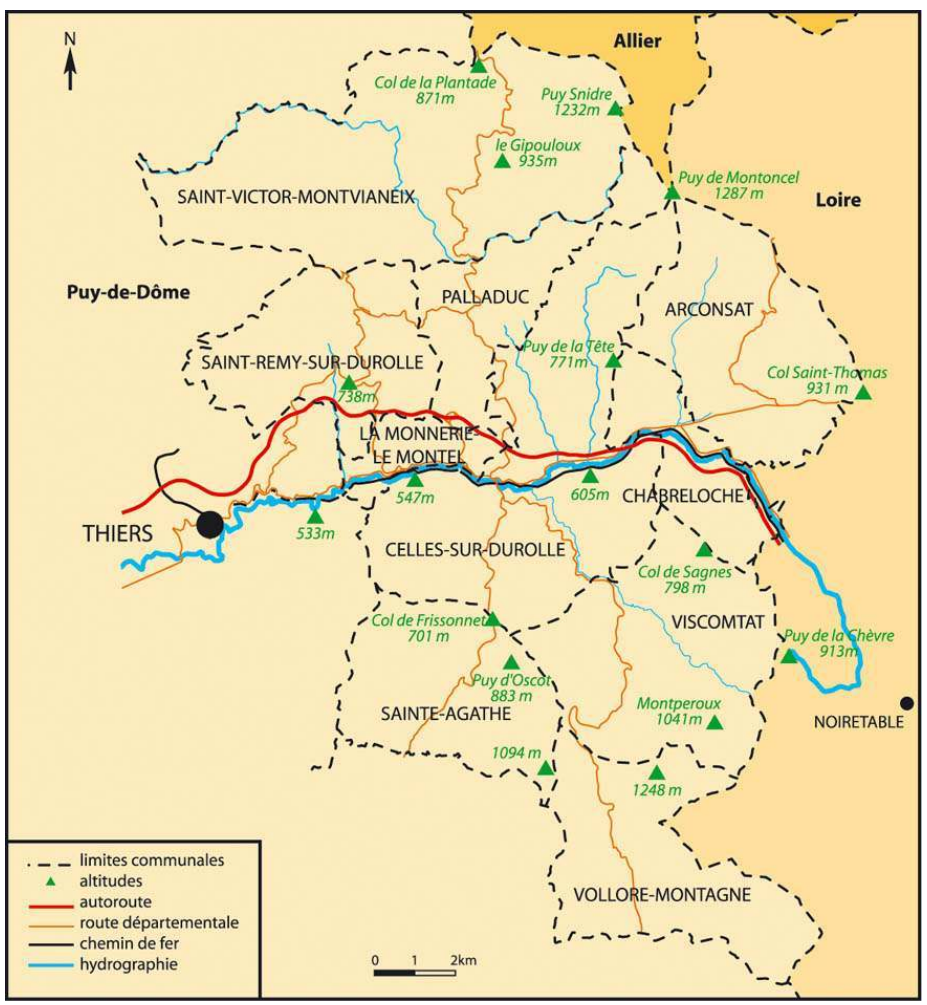

Carte de situation de l'aire de recensement

Beauparland-Dupuy, Guylaine (c) Inventaire général, Région Auvergne, ADAGP, 2007

Dans un premier temps, il avait été envisagé de ne repérer que les " ateliers à domicile dans lesquels du matériel était trouvé » mais l'opération a été ré-orientée de manière à plutôt mettre en évidence l'impact sur le paysage d'une telle organisation du travail et à établir une typo-chronologie.

\section{Prendre la mesure d'un phénomène et établir une typo-chronologie}

Les Statistiques industrielles de 1875 pour l'Auvergne mentionnent l'existence de deux types d'ouvriers à domicile : ceux de la région de Thiers qui travaillent dans la coutellerie, et les ouvrières de la dentelle de la région d'Ambert, Arlanc et Viverols ${ }^{3}$. Afin de donner une idée de l'ampleur du phénomène coutelier à cette date, les chiffres sont éloquents : 22000 ouvriers sont recensés, tous confondus, ouvriers en usine et ouvriers à domicile, mais il est spécifié que " parmi eux, beaucoup s'occupent aussi des travaux des champs ». A côté d'eux, « seulement » 5500 ouvrières fabriquaient de la dentelle ${ }^{4}$.

4 Notons que c'est par la double occupation (coutellerie et travaux des champs) que les ouvriers à domicile sont distingués des ouvriers d'usines, mais ils sont comptabilisés de manière, malheureusement, moins précise (« beaucoup » sur 22000 !).

5 A titre de comparaison à l'échelle nationale, les chiffres des Statistiques industrielles de 1811 donnent 6185 ouvriers dans la coutellerie de la région de Thiers, dont 2000 travaillent isolément, tandis qu'à la même période (1814) le Jura ne compte que 1032 
ouvriers dans la tournerie-tabletterie (pipes de Saint-Claude, etc) 5 . Ces industries sont très comparables car, comme en Montagne thiernoise, les ouvriers de la région de SaintClaude « contribuent à développer une double activité : travaux agricoles pendant la belle saison et activité artisanale en famille de novembre à avril ».

Si en 2007 les seuls ouvriers à domicile en Auvergne sont encore les "émouleurs, polisseurs et trempeurs de la région de Thiers $»^{6}$, auxquels s'ajoutent les tisseurs de soie et les tisseurs sur métiers mécaniques (Puy-de-Dôme et Haute-Loire) ainsi que les rubaniers de Haute-Loire, la pratique est cependant en voie de disparition. C'est en partie pour répondre à cette situation d'urgence créée par les mutations, transformations et destructions qui mettent en péril le patrimoine lié à cette "industrie à domicile » que la Région s'est rendue partenaire d'une opération de recensement de ses derniers vestiges.

7 Sur place, le phénomène est bien connu. Toutes les maisons du territoire concerné sont réputées avoir possédé leur atelier, qu'il s'agisse de clouage, de montage, d'émouture, de forge ou de polissage. Cette assertion a constitué l'hypothèse simple à partir de laquelle nous avons construit notre recensement : il fallait vérifier dans quelle mesure le paysage bâti actuel, urbain et rural, en répercutait l'écho.

8 La méthode des services de l'Inventaire général du patrimoine culturel a été appliquée, destinée à organiser en quelque sorte un phénomène connu, à le quantifier et tenter de le cerner historiquement. Il en ressort un état des lieux et une typo-chronologie qui pourront servir aux collectivités partenaires, Conseil régional, Communauté de communes de la Montagne thiernoise et commune de Saint-Rémy-sur-Durolle, tant du point de vue de la connaissance du territoire que du tourisme. En effet, s'agissant de désigner clairement aux visiteurs occasionnels une partie de l'histoire de la région, cette problématique industrielle devient incontournable.

\section{Lire l'industrie à domicile depuis l'espace public, qualifier le paysage}

L'ampleur du sujet a nécessité d'en définir certains indicateurs. Trouver les moindres traces du travail à domicile était une entreprise trop vaste pour être mise en œuvre de manière raisonnable, dans un temps donné : la visite intérieure de toutes les maisons, jointe à une enquête orale systématique, aurait été la seule manière d'informer exhaustivement le sujet, à la réserve près que l'impossibilité de vérifier les données recueillies oralement aurait constitué une limite importante à l'exercice ${ }^{7}$. Par exemple, le clouage des couteaux ne nécessite de disposer que d'un coin de table. Par ailleurs, aucune source d'archives directement utilisable ne permet de localiser les ouvriers à domicile, ceux-ci n'étant pas assujettis à la patente. En effet, le dépouillement des carnets de patentes pour l'année 1912 ne mentionne aucun fabricant de couteaux sur les communes d'Arconsat (où l'on dénombre 11 scieries, un moulin à moudre et un layetier) et SainteAgathe (où l'on ne mentionne que deux exploitants de moulins), tandis qu'à Saint-Rémysur-Durolle 43 fabricants couteliers "non expéditeurs» et 18 fabricants couteliers « expéditeurs » sont recensés (représentant un total de 384 ouvriers), ${ }^{8}$, or le statut de l'ouvrier à domicile est bien différent de celui de l'artisan, a fortiori de celui du patron d'entreprise. 
Figure 2

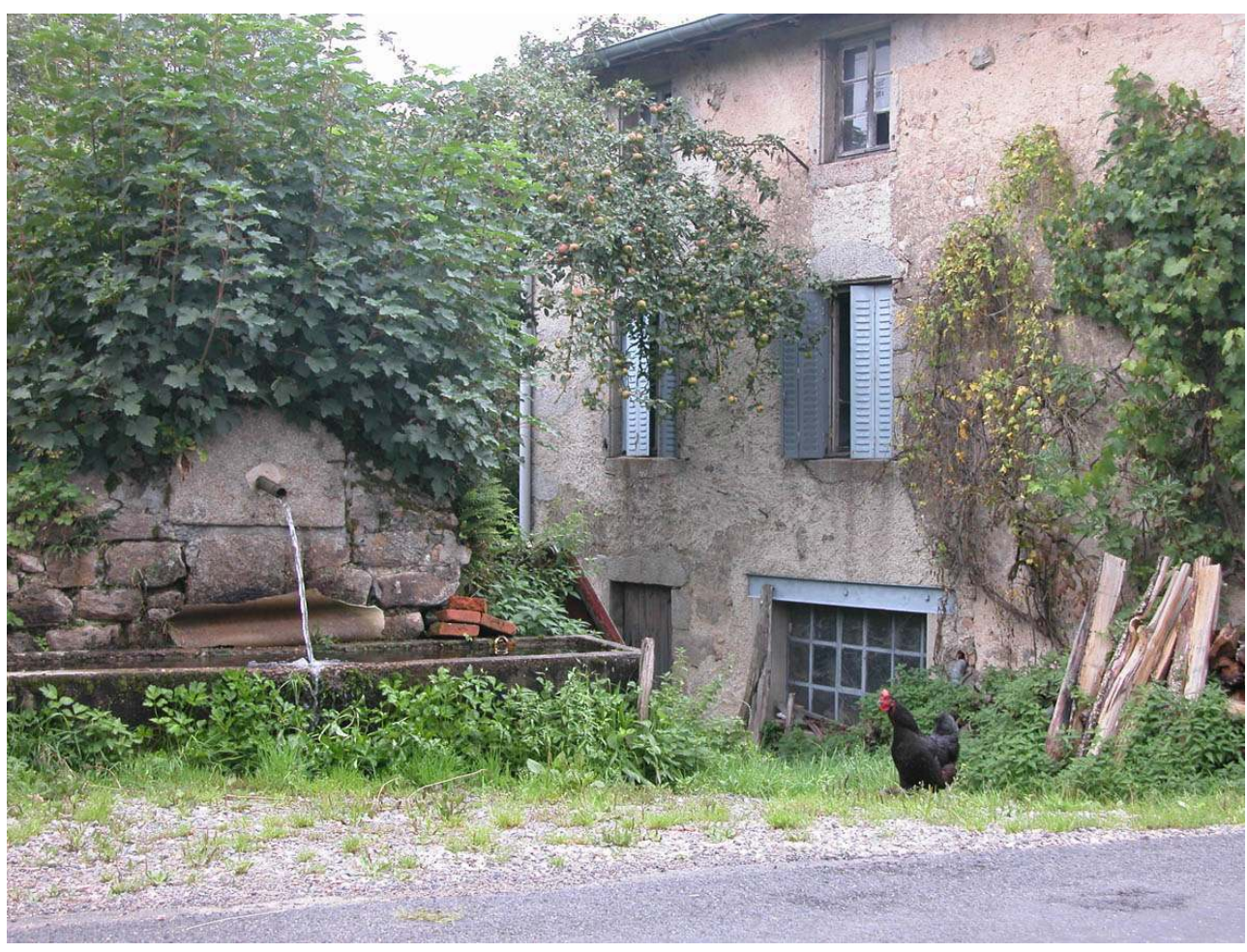

La Courtade, commune de Viscomtat. Fenêtre d'atelier dans l'étage de soubassement de la maison Renaud, Bénédicte @ Inventaire général, Région Auvergne, ADAGP, 2007

10 Nous avons choisi de croiser deux objectifs : connaissance du patrimoine industriel et connaissance du paysage. Un critère essentiel a donc été retenu qui permet de lire «l'industrie à domicile » depuis l'espace public (fig. $\mathbf{n}^{\circ}$ 2). La fenêtre d'atelier, localement dénommée « vanne » a en effet constitué l'indice discriminant qui pouvait être localisé et quantifié. Sa forme a évolué : à condition d'être associé à d'autres éléments, l'indice pouvait aussi servir de critère de datation. En prenant ce parti, nous avons réaffirmé que le premier document sur l'objet d'étude, c'est l'objet-même, conservé in situ. En outre, l'inscription récurrente de cet indice dans le bâti courant, le désigne comme un marqueur fort du paysage de la montagne thiernoise, pour qui le visite occasionnellement ${ }^{9}$.

11 Les limites de ce choix résident dans la possibilité que les ateliers éclairés par ces fenêtres ne sont pas tous des ateliers où l'on a travaillé les couteaux (ou les tire-bouchons, ou les rasoirs, ou les "flammes $»^{10}$, ou les machettes ${ }^{11} \ldots$..). A la marge, il pourra s'agir, par exemple, d'ateliers de menuisiers ${ }^{12}$, de mécanique générale ${ }^{13}$ ou de bouchers (encore que des témoignages allant dans ce sens ne nous assurent pas de la destination initiale de l'atelier) ${ }^{14}$. Par ailleurs, les structures complètes ont été privilégiées : la fenêtre d'atelier privée de ses petits carreaux, autrement dit la présence seule d'un linteau caractéristique, n'a pas été retenue dans le recensement, sauf lorsque l'indice incomplet mais isolé pouvait constituer une réponse à la question de l'implantation des ateliers sur le territoire.

12 Il a été parfois difficile de trancher entre atelier et usine : quand le gabarit du bâtiment s'apparentait à celui des habitations et bâtiments environnants, il a été retenu. 
Dans un site aussi indiscutablement marqué par la présence des ateliers, les erreurs commises ne peuvent pas avoir eu d'influence significative sur l'analyse qui découle des cartes de recensement.

Figure 3

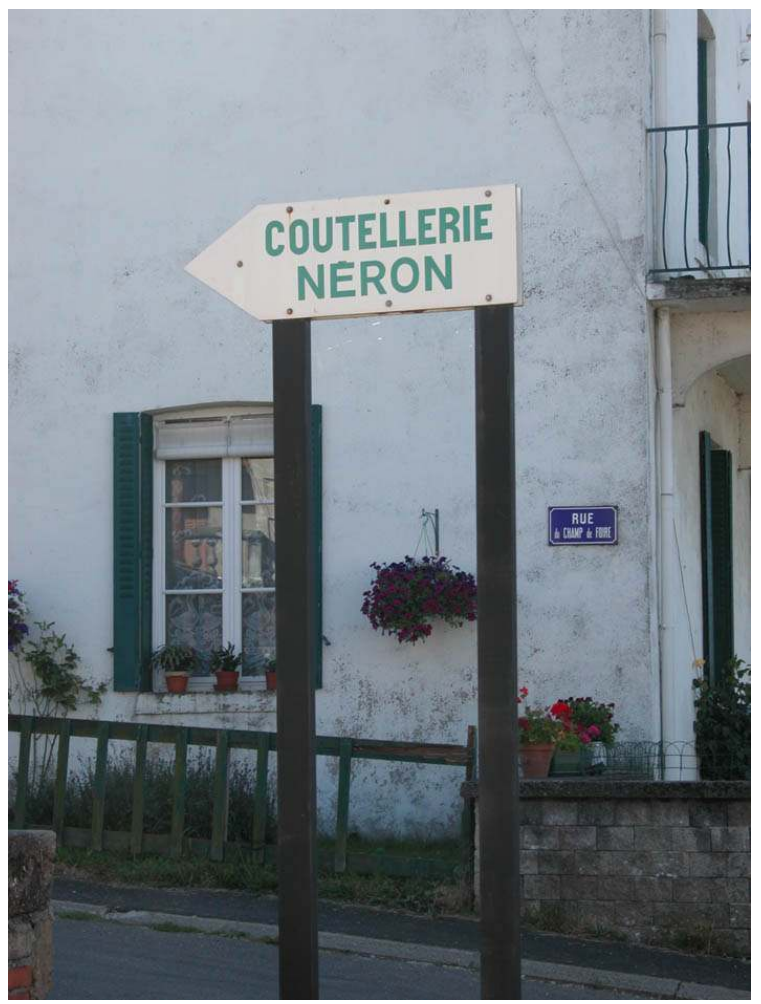

Chabreloche, bourg. Enseigne

Renaud, Bénédicte (c) Inventaire général, Région Auvergne, ADAGP, 2007

Il a semblé par ailleurs intéressant de signaler la présence d'enseignes et pancartes indicatrices d'ateliers lorsqu'elles jouaient le rôle d'édicules-repères dans le paysage (fig. $\mathbf{n}^{\circ}$ 3). Pour le visiteur occasionnel, elles contribuent à expliciter en quelque sorte l'ensemble des indices visibles de l'espace public ${ }^{15}$.

\section{Une vue d'ensemble bien ancrée dans le territoire}

Il arrive que le constat dressé par les historiens à partir des textes et d'un choix de sites a priori, ajouté à la tradition orale véhiculée par les habitants ne suffisent pas à une juste connaissance des choses. C'est ainsi que les chercheurs de l'Inventaire général se sont fixé de parcourir tout le territoire ${ }^{16}$ et que les données collectées ont été systématiquement cartographiées (le fonds de référence étant celui de l'I.G.N., au 25 000e). Il s'est agi de répondre à une double exigence d'exhaustivité : au niveau de l'enquête ${ }^{17}$ et au niveau de la restitution. Cet inventaire a été reporté sur un Système d'information géographique, duquel nous avons tiré les cartes qui illustrent notre article ${ }^{18}$. Nous avons pu donner une image claire et fiable de l'implantation sur le territoire des ateliers encore perceptibles depuis l'espace public.

La photographie "de travail », jointe à chacun des ateliers recensés, sans avoir la qualité des photographies professionnelles, s'affiche comme une trace patrimoniale et donc 
comme une justification de la donnée rapportée sur la carte. «L'image, surtout quand elle est systématique, est porteuse d'une grande quantité d'informations qui vaut souvent plus qu'une description, par définition, exclue du recensement $»^{19}$. Cependant, un photographe du service a été dépêché sur certains sites particulièrement intéressants ou menacés.

\section{Dénombrement, statistiques et cartographie}

1123 ateliers (disposant d'une ou plusieurs fenêtres caractéristiques) ont été recensés qui impriment leur marque sur le paysage ${ }^{20}$.

Pour la commune de Celles par exemple, jugée la plus représentative, sur 760 résidences principales recensées en 1999, auxquelles il faut ajouter 87 résidences secondaires, 235 ateliers ont été repérés : $27,7 \%$ des habitations témoignent encore sans équivoque d'un travail à domicile, ce qui semble peu au regard de la tradition qui veut que «toutes les maisons abritaient une activité liée au couteau » mais ce qui est beaucoup compte tenu du fait que d'une part, certaines maisons ne disposaient pas d'ateliers proprement dit et d'autre part, que le travail à domicile a presque complètement disparu. Bien sûr, ce pourcentage n'est pas le reflet d'une réalité historique, c'est-à-dire qu'il serait faux de décréter que seulement $28 \%$ des habitations possédaient leur atelier, il est le reflet d'une situation qui rend compte des aléas de la conservation, surtout dans le cas où l'atelier est indépendant de la maison car on peut supposer que les fenêtres d'atelier moins fréquemment intégrées au volume de la maison disparaissent.

En revanche, la cartographie est parlante sur les zones d'implantation des ateliers: quasiment tous les hameaux ou écarts du territoire sont concernés. Ceux qui ne le sont pas sont soit situés à la marge du territoire (Le Sopt, Lodigerie, Archimbaud, commune de Vollore-Montagne, par exemple), là où l'agriculture suffit à la subsistance, soit des cas isolés (La Jalerie, commune de Vollore-Montagne ; Le Vernet, commune de Celles) pour lesquels l'hypothèse pourrait être posée d'anciens fiefs puisque ce sont dans ces lieux-là que l'on repère les rares vestiges d'une architecture qui adopte les codes d'une classe sociale aisée (mur de clôture de la parcelle ou bien croisées avec arêtes chanfreinées, etc).

Des hameaux comme La Gonie (commune de Sainte-Agathe) ou Montbout (commune de Vollore-Montagne), ne disposent plus d'aucun indice, même incomplet: seuls les témoignages oraux peuvent faire état d'une activité coutelière ancienne mais, dans ce cas, aucun report sur la carte n'a été fait. De même des éclats de fer sur un mur, indiquant pourtant l'emplacement d'une ancienne forge, n'ont pas été retenus.

21 Hors la structure administrative de la Communauté de communes de la Montagne thiernoise, même si l'on y joint les limites communales de Saint-Rémy, le recensement des ateliers pourrait être poursuivi : au nord par exemple, dans le canton de Châteldon, la catégorie des maisons avec atelier de monteur à domicile se distingue, là aussi, encore nettement ${ }^{21}$, ou bien dans la commune de Vollore-Ville ${ }^{22}$. Le recensement pourrait dès lors inclure toutes les zones limitrophes de manière à dresser la carte générale des ateliers d'ouvriers à domicile liés à l'activité coutelière de la région de Thiers. 


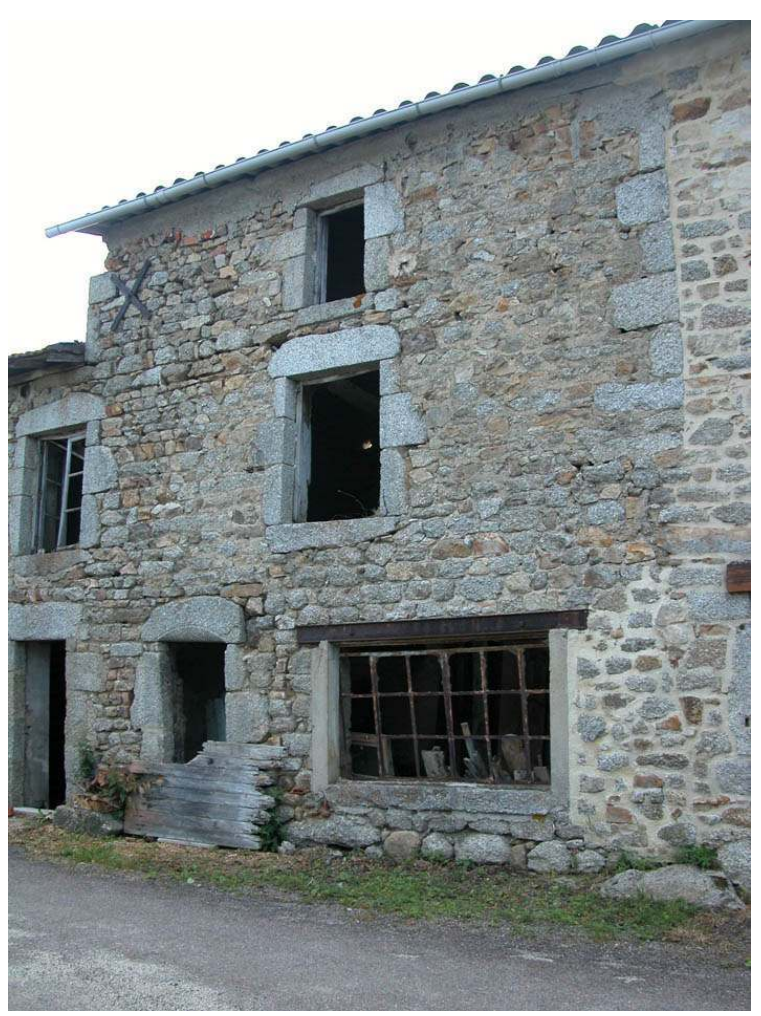

Chouvel, commune de Saint-Rémy-sur-Durolle. Fenêtre d'atelier au rez-de-chaussée de la maison Ceroni, Brigitte ( I Inventaire général, Région Auvergne, ADAGP, 2007

Une telle opération de recensement reste un outil irremplaçable pour la connaissance d'un phénomène patrimonial original et elle permet aussi de sensibiliser la collectivité gestionnaire à l'opportunité qu'il y aurait à encourager la préservation de ces vestiges identitaires (fig. $\left.n^{\circ} 4\right)$.

\section{La typo-chronologie des ateliers}

Une première phase du recensement a consisté à valider la répartition en catégories d'ateliers qui nous avait été proposée (atelier en rez-de-chaussée, accolé ou isolé). Nous avons organisé le test à l'échelle de la commune de Celles réputée la plus représentative. Des amendements ont été apportés pour la suite du recensement.

Une typologie a donc été établie en fonction de critères de situation par rapport à la maison; nous avons distingué les ateliers intégrés à l'habitation (en rez-de-chaussée ou à l'étage) des ateliers intégrés aux bâtiments agricoles, et de ceux simplement accolés à la maison, ou encore de ceux qui sont indépendants (sur un même fonds ou à proximité de l'habitation). 


\section{Type 1 : atelier au rez-de-chaussée ou en soubassement de la maison} soit $34,9 \%$ (fig. $\mathbf{n}^{\circ}$ ) ).

Figure 5

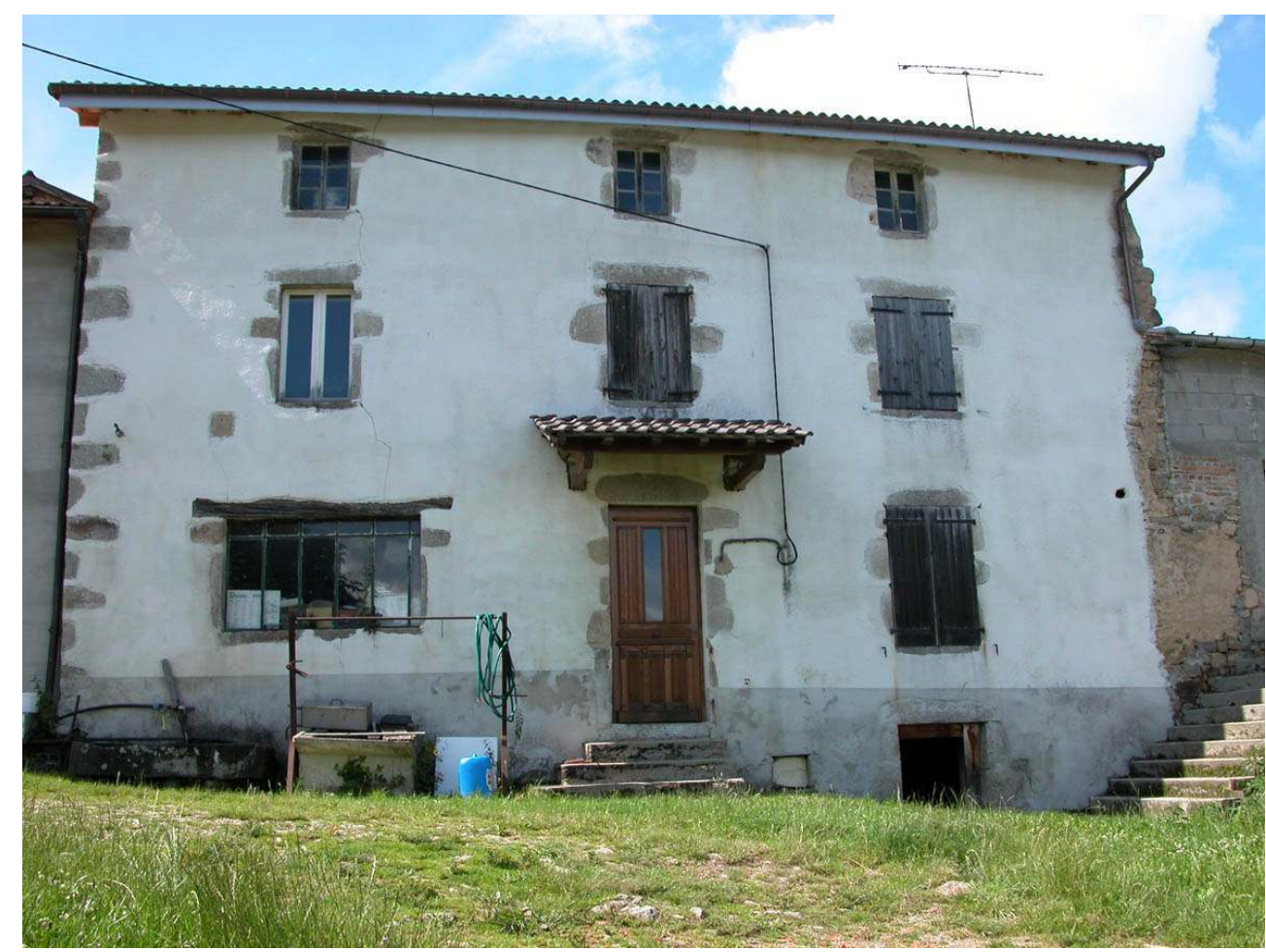

La Faye, commune de Celles-sur-Durolle. Fenêtre d'atelier au rez-de-chaussée de la maison, perturbant le système des travées

Renaud, Bénédicte (c) Inventaire général, Région Auvergne, ADAGP, 2007

Il correspond souvent, semble-t-il, à l'atelier installé dans la pièce principale de l'habitation, qui fait le plus souvent office de cuisine. Un coin de cette salle, juste derrière la grande fenêtre, était aménagé avec une large et longue tablette de bois, sorte d'établi où l'on s'installait pour le montage des couteaux. Parfois, une pièce spécifique était réservée à cette activité : même situation de la baie en façade, mais organisation plus précise à l'intérieur, chaque fonction bénéficiant d'un lieu différencié. C'est un type d'ateliers qui se retrouve indifféremment dans les écarts ruraux et dans les gros bourgs (il ne nécessite pas de parcelle de terrain adjointe à l'habitation). Il est permis de penser que ce type d'organisation est l'un des premiers (peut-être avec le quatrième présenté cidessous) à s'être mis en place, témoignant de façon assez nette du mélange des travaux ici domestiques, plus loin paysans - et ouvriers à l'intérieur d'un même espace.

Ces « vannes » en rez-de-chaussée de maisons ont souvent été transformées, du moins en partie, une fois l'activité coutelière abandonnée : il a été facile de les réutiliser en grandes baies de cuisine ou de salle à manger, leur côté artisanal étant gommé aisément par l'adjonction de volets, de nouveaux petits bois de fenêtres et de rideaux... Seules leurs dimensions particulières dans l'alignement des travées permettent alors de les repérer. 
Signalons un cas de figure (un précurseur ?), à Mondière (commune de Viscomtat) où la fenêtre est à peine plus large que celle qui la surmonte mais où l'aménagement intérieur et le matériel subsistants dissipent les doutes quant à la destination de la pièce éclairée, en rez-de-chaussée.

\section{Type 2 : atelier indépendant de la maison, sur la même parcelle ; atelier isolé}

Ce type arrive, par le nombre de représentants, en deuxième position avec 287 éléments sur 1123 , c'est-à-dire $25,6 \%$ (fig. $\mathbf{n}^{\circ}$ 6).

Figure 6

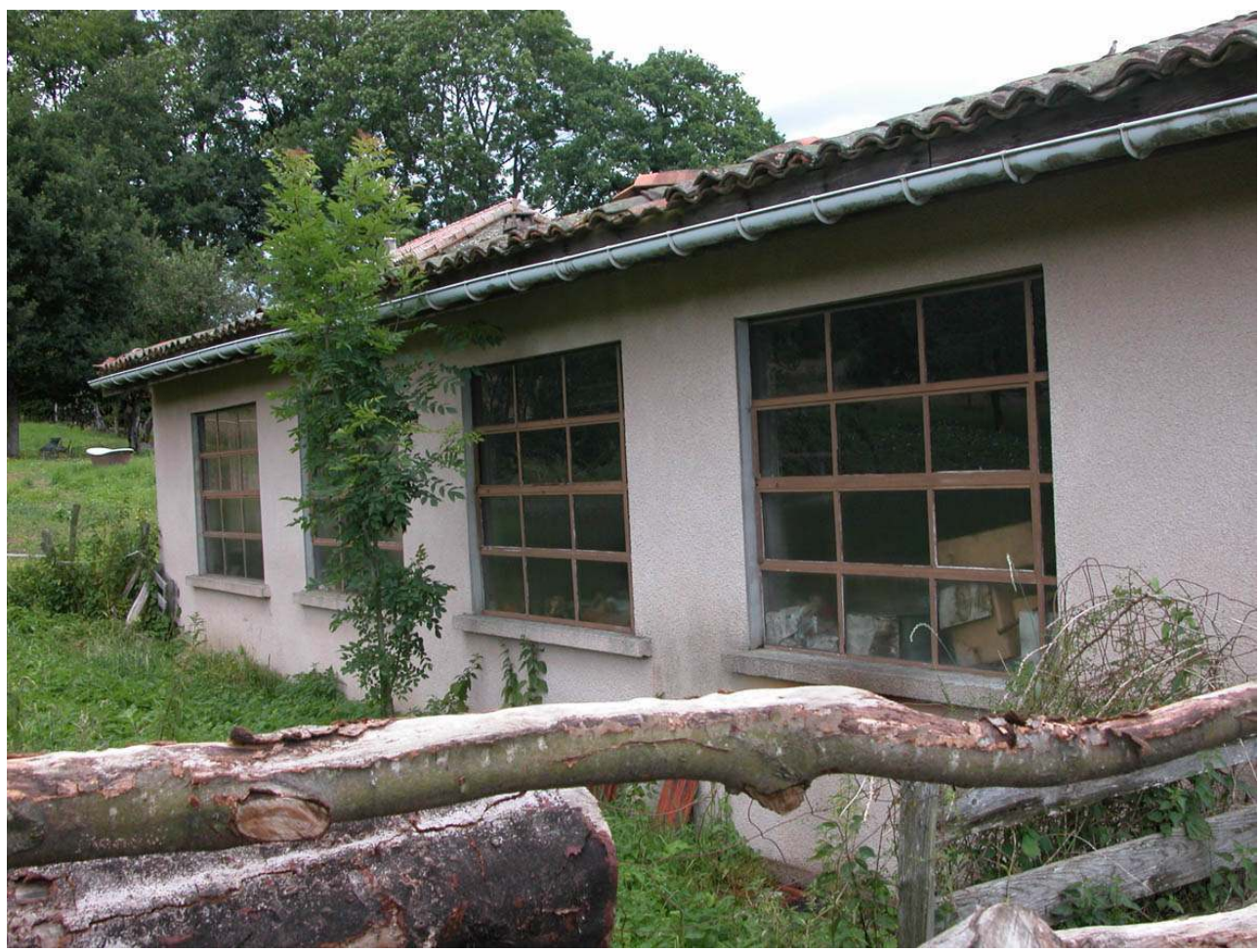

La Vernière, commune de Celles-sur-Durolle. Atelier isolé (spécialisé dans les tire-bouchons)

Renaud, Bénédicte (c) Inventaire général, Région Auvergne, ADAGP, 2007

Il se subdivise cependant en deux branches : les ateliers indépendants de l'habitation mais installés sur la même parcelle, en fond de terrain ou sur le côté par exemple; les ateliers complètement isolés, qui ne sont pas directement reliés à une habitation. Ces deux sousensembles ont été malgré tout regroupés dans un même type car ils présentent les mêmes formes architecturales, quelle que soit leur position « foncière ». Ce sont dans la majorité des cas des constructions d'un seul niveau (même si l'on trouve des ateliers possédant un deuxième niveau - avec des fenêtres ordinaires cependant); ces rez-de-chaussée sont éclairés par une ou plusieurs « vannes » selon leur importance, l'avantage de leur position isolée étant la possibilité de percer de baies plusieurs murs pour un éclairage maximal quelle que soit l'orientation ou l'heure de la journée. Les toitures sont à un pan pour les ateliers les plus simples, mais le plus souvent à deux pans dès qu'ils prennent un peu d'importance. L'atelier, dans sa forme la plus modeste, est comparable à une cabane de jardin. 
Il s'agit d'un type de construction qui s'est particulièrement multiplié pendant la période florissante de la coutellerie en Montagne thiernoise (voir le paragraphe sur la chronologie). Ce lieu de travail isolé, contrairement au précédent, dénote déjà une part plus importante donnée à l'activité coutelière, bien séparée des autres travaux. Ces ateliers indépendants sont d'ailleurs parfois perçus et décrits par leurs propriétaires comme de petites « usines » où l'on se déplace (à un ou plusieurs ouvriers) pour aller au travail.

\section{Type 3 : atelier en rez-de-chaussée accolé à la maison, sur le côté ou à l'arrière}

31 Ce type d'atelier, en troisième position, représente $24,7 \%$ de notre corpus dans son ensemble (c'est-à-dire 277 éléments sur 1123 recensés) (fig. $\mathbf{n}^{\circ} 7$ ).

Figure 7

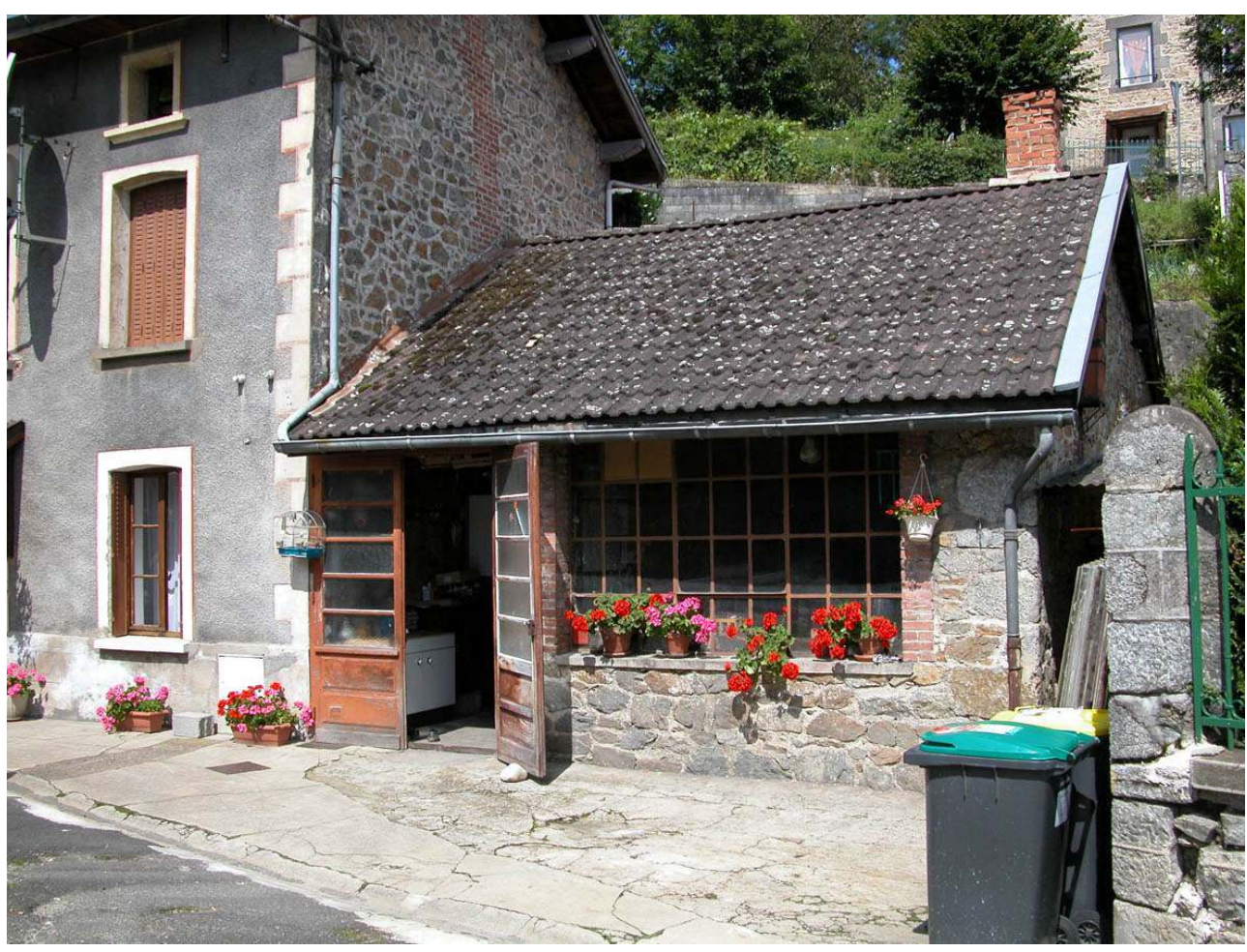

La Monnerie-le-Montel, bourg (atelier de trempe). Atelier accolé à la maison

Renaud, Bénédicte (c) Inventaire général, Région Auvergne, ADAGP, 2007

Sans doute représentatifs de l'étape intermédiaire entre le premier et le deuxième des types présentés ci-dessus, ces ateliers ont pu venir s'accoler à la maison lors d'un nécessaire agrandissement de l'espace d'habitation et prendre parfois le relais d'ateliers du type "en rez-de-chaussée d'habitation ", l'espace réservé jusqu'alors au premier retrouvant sa destination de salle commune. Il semble plus rare qu'un atelier contemporain de l'habitation ait été construit sous cette forme et accolé dès l'origine...

Ici, l'activité coutelière est déjà distincte des autres mais le lien existe encore avec le logis ; l'entrée se fait la plupart du temps par une porte extérieure propre à l'atelier luimême, mais il arrive qu'elle se fasse directement depuis la maison. 

chaussée, de petite taille, couverte d'un toit en appentis, et présentant un nombre restreint de « vannes ».

\section{Type 4 : atelier combiné ou accolé à un bâtiment agricole}

Sur 1123 éléments, 146 appartiennent à cette famille d'ateliers, soit $13 \%$ (fig. $\mathbf{n}^{\circ}$ 8).

Figure 8

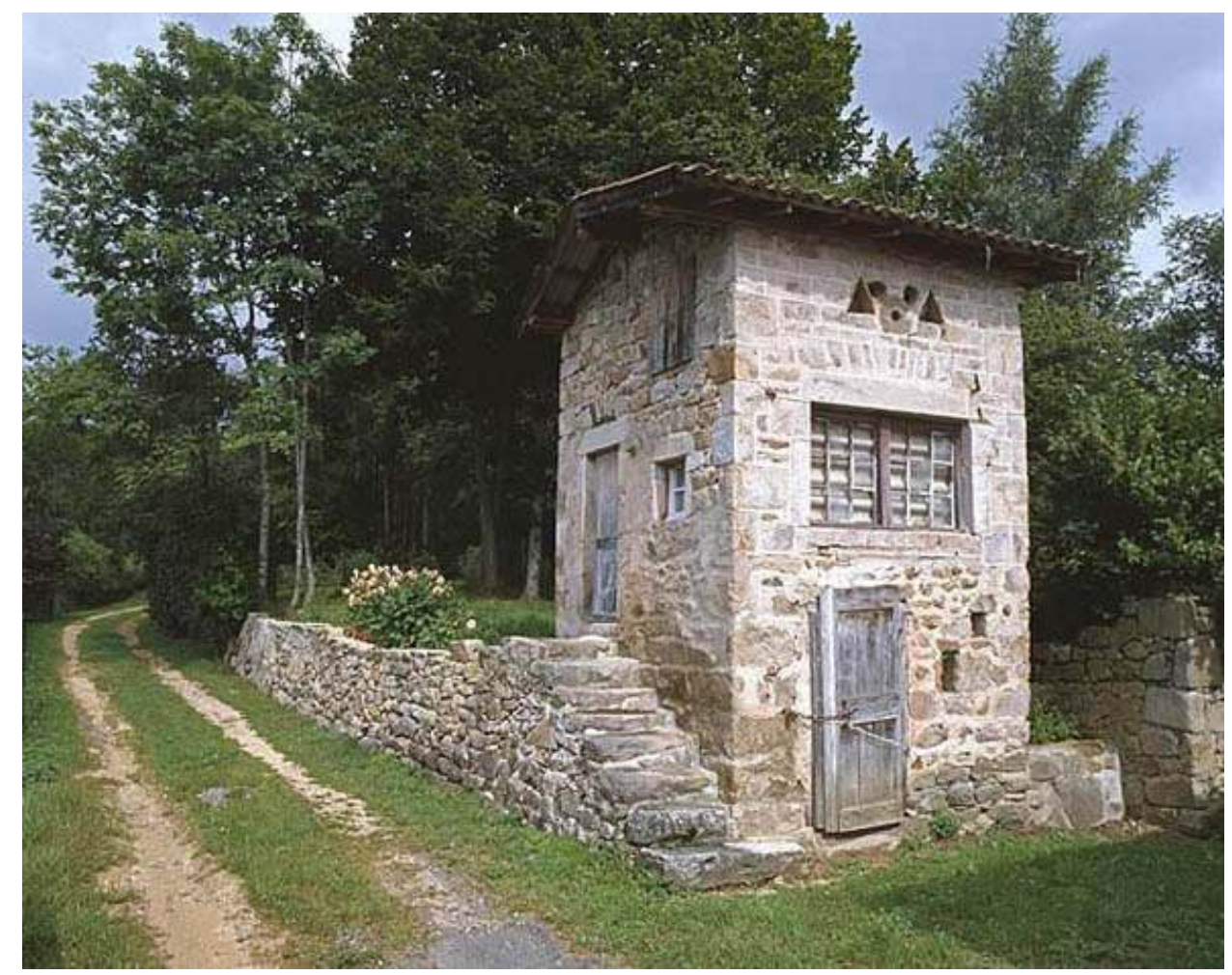

La Pauze, commune d'Arconsat. Atelier intégré dans une dépendance agricole composée de soue, atelier et pigeonnier

Périn, Jean-Michel (C) Inventaire général, Région Auvergne, ADAGP, 2007

Ils ont été bien évidemment recensés dans les zones rurales, qui représentent une grande partie du territoire de la Communauté de communes.

37 Il est intéressant de voir comment l'architecture vernaculaire agricole s'est aisément adaptée en Montagne thiernoise à cette fonction complémentaire que représentait l'activité coutelière; les traditionnelles «fermes en maison-bloc» auvergnates (dont le logis et les parties agricoles sont regroupées sous un même toit), les granges-étables, et même, ce qui est peut-être le plus frappant, les petits bâtiments annexes de l'activité paysanne, tels que porcheries, séchoirs ou pigeonniers, tous ont intégré de façon naturelle les ateliers et leurs baies caractéristiques.

Dans le cas des fermes et des granges-étables, la solution couramment adoptée a été de percer la « vanne » à l'emplacement attendu de la petite fenêtre d'étable, l'atelier venant s'installer en enclave dans cet espace ; il peut également avoir été placé de l'autre côté de la porte de grange et faire ainsi pendant à l'étable. 

d'ajouter une fonction supplémentaire à l'ensemble. C'est ainsi que l'on trouve des ateliers sur porcherie, des séchoirs sur atelier, des ateliers-fours à pain, des pigeonniersateliers, voire une composition regroupant porcherie en rez-de-chaussée, atelier au 1er étage (avec accès par un escalier extérieur) et pigeonnier en comble... En somme, de multiples variations ont été imaginées à partir de constructions déjà bien implantées dans les campagnes, pour installer, au gré des besoins, un peu de vie ouvrière au cœur de la vie agricole.

\section{Type 5 : atelier à l'étage, dans une dépendance accolée}

Seuls 16 ateliers sur 1123 , soit 1,4\%, appartiennent à cette catégorie (fig. $\mathbf{n}^{\circ} \mathbf{9}$ ).

Figure 9

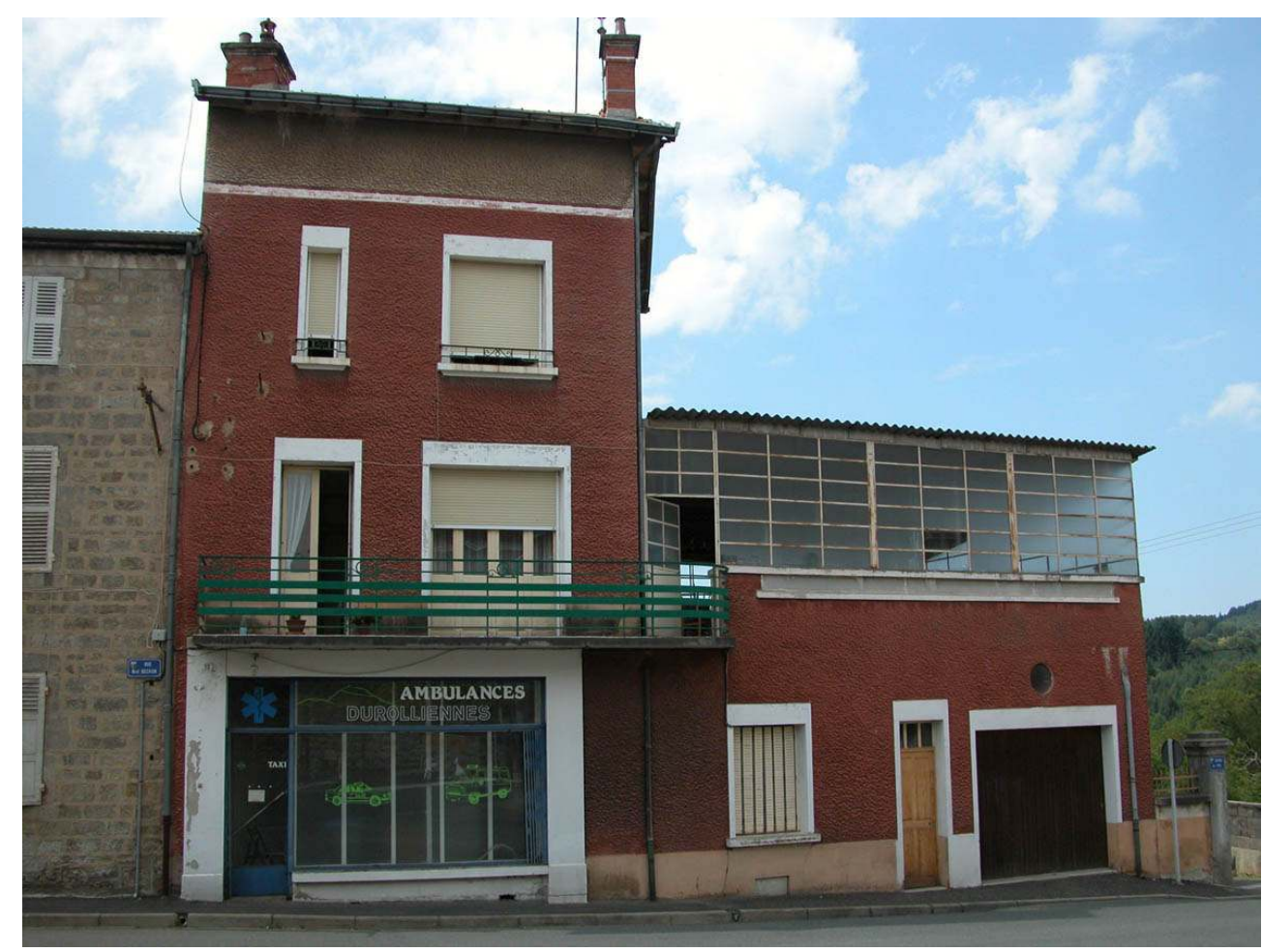

Saint-Rémy-sur-Durolle, bourg. Atelier accolé et en étage

Ceroni, Brigitte (c) Inventaire général, Région Auvergne, ADAGP, 2007

41 Il est difficile de déterminer dans quelles circonstances ces ateliers en étage, en très petit nombre, ont été installés: simple surélévation due aux circonstances, aménagement prévu ainsi dès l'origine, ou combinaison de plusieurs espaces artisanaux superposés - à l'image des rouets...

Ce type pourrait, dans l'absolu, être rattaché au type 3 (« en rez-de-chaussée accolé à la maison ») et considéré comme un sous-ensemble ou une variante, de celui-ci. 


\section{Type 6 : atelier à un étage de la maison}

43 Il s'agit là, très nettement, du type le moins représenté de tous, avec seulement 5 spécimens (fig. $\mathbf{n}^{\circ} \mathbf{1 0}$ ).

Figure 10

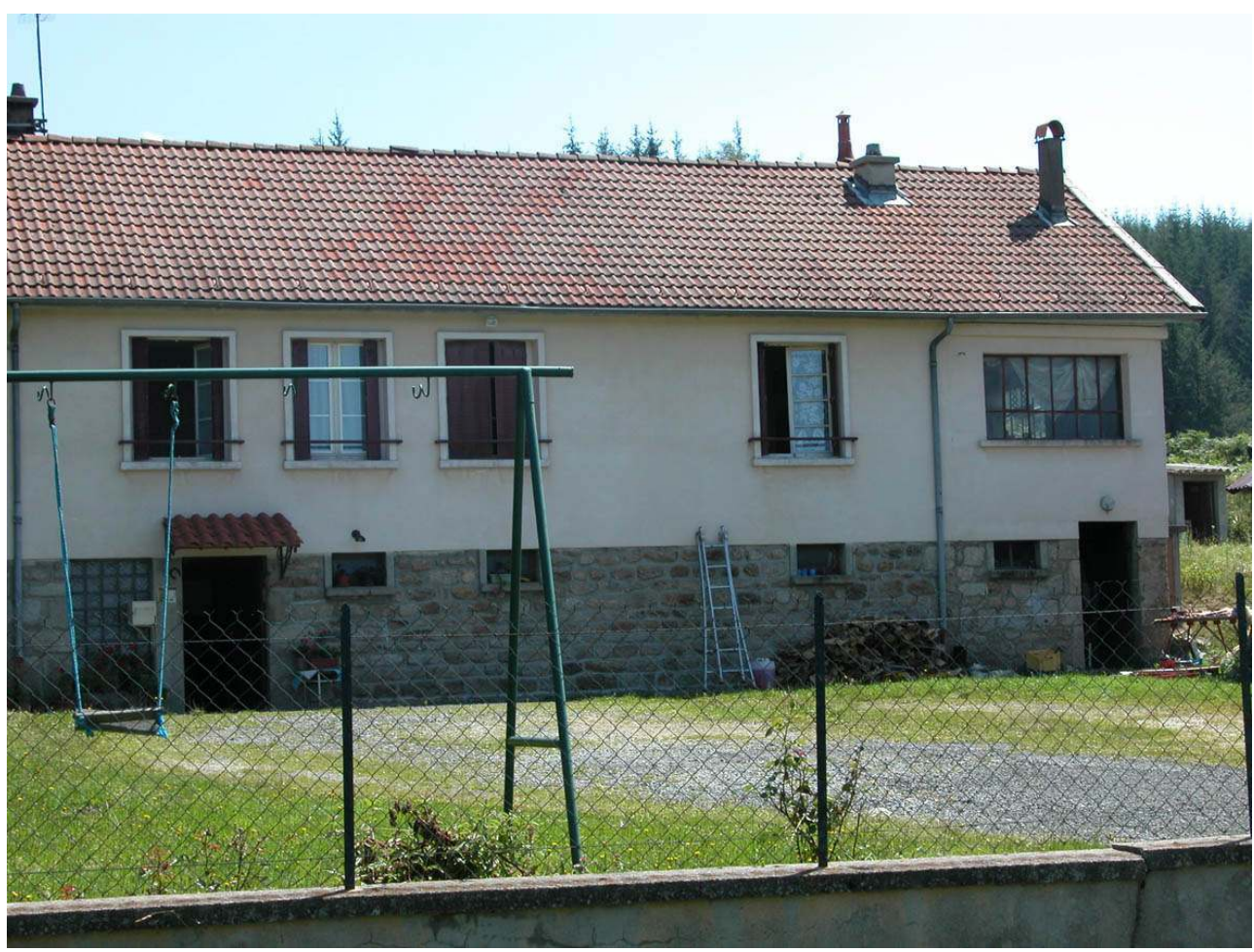

La Loge, commune de Vollore-Montagne. Atelier intégré dans la maison et en étage

Renaud, Bénédicte @ Inventaire général, Région Auvergne, ADAGP, 2007

44 Là encore, le très petit nombre d'éléments de ce type ne permet pas de conclusions statistiques pertinentes, et semble plutôt être un avatar des ateliers intégrés à l'habitation (voir type 1 ci-dessus).

\section{Le classement chronologique}

La tentative de classement chronologique des différents ateliers recensés s'est appuyée essentiellement sur les observations liées aux matériaux, aux formes et à la mise en oeuvre des édifices situés dans les zones voisines étudiées par l'Inventaire précédemment ${ }^{23}$. Un nombre, même réduit, d'ateliers datés de l'aire d'étude a suffi à confirmer l'échelle de référence ${ }^{24}$. Il est arrivé que le témoignage oral se substitue aux dates portées. Ces tranches chronologiques ont été croisées avec des césures liées à l'histoire de la coutellerie thiernoise $e^{25}$. Les ateliers ont donc été classés en cinq grandes périodes de construction : période 1 allant du XVIII ${ }^{\mathrm{e}}$ siècle jusqu'au milieu du XIX ${ }^{\mathrm{e}}$ siècle ; période 2, de la deuxième moitié du XIXe siècle jusqu'à 1918; période 3 correspondant à l'entredeux-guerres; période 4, couvrant les années 1945 à la fin des années 1960 ; et enfin, période 5, de 1970 à nos jours. 


\begin{abstract}
peuvent avoir évolué de façon variable d'un endroit à l'autre, l'un utilisant les dernières avancées techniques, alors qu'au même moment l'autre a préféré des méthodes depuis longtemps éprouvées. Des incertitudes quant à la datation subsistent aussi forcément quand il s'agit d'ateliers qui ne sont pas construits par des professionnels du bâtiment, mais elles ne peuvent mettre à mal le classement général.
\end{abstract}

césures chronologiques peuvent fluctuer de quelques années, les façons de construire

\title{
Période 1 : du XVIII siècle jusqu'au milieu du XIXe siècle
}

\section{Figure 11}

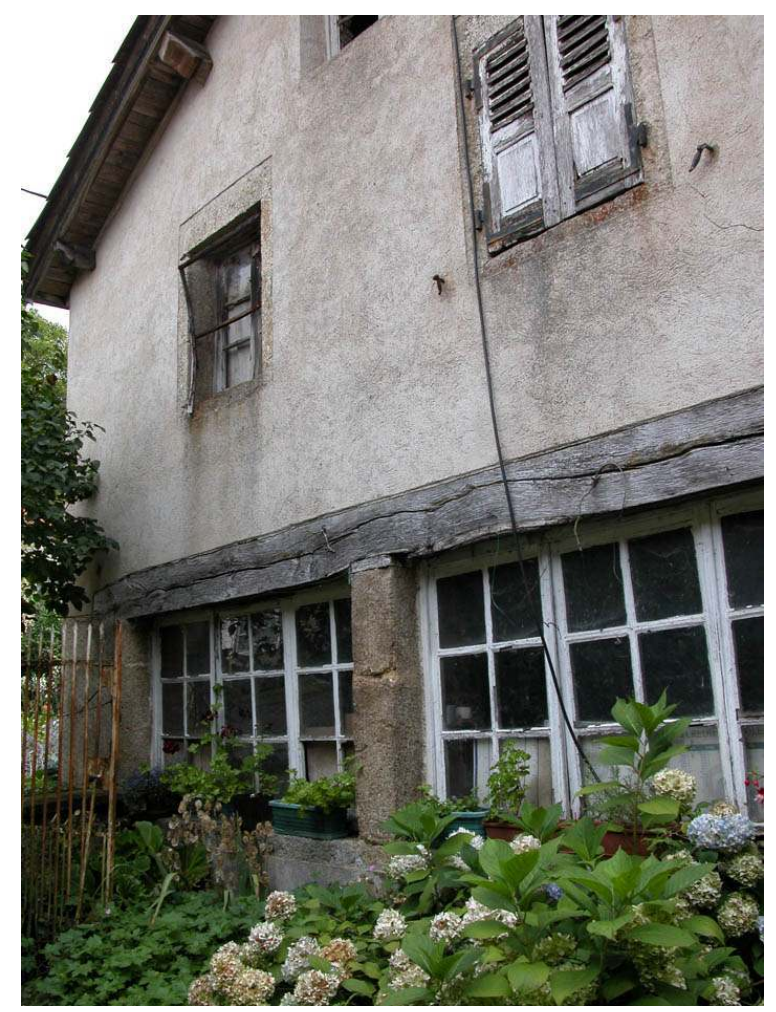

Pubereau, commune de Palladuc. Fenêtre d'atelier ancienne avec petits bois Ceroni, Brigitte (c) Inventaire général, Région Auvergne, ADAGP, 2007

Elle se signale par une utilisation fréquente du bois pour les encadrements ; les fenêtres d'ateliers sont alors d'une forme assez éloignée de ce que l'on trouvera au milieu du XX siècle (fig. $\mathbf{n}^{\circ}$ 11). Ces baies viennent s'insérer dans des murs en moellons de pierre, et voisinent avec d'autres ouvertures en bois également, bien que d'un format différent. Encore relativement peu développées en hauteur mais étirées en longueur et donc souvent recoupées par des «trumeaux » intermédiaires séparant ainsi la fenêtre en deux ou trois parties, les plus anciennes ouvertures (remontant vraisemblablement au XVIII ${ }^{\mathrm{e}}$ siècle, voire un peu au-delà) occupent une assez large surface du niveau qu'elles éclairent. Certains ateliers ont même toutes leurs baies (porte et fenêtres) regroupées sous un même linteau, ce qui semble former le modèle le plus simple et le plus modeste. On trouve aussi parfois (plus on avance dans le temps semble-t-il) la combinaison de la pierre et du bois pour les encadrements (linteau et piédroits d'un matériau différent), moins 
souvent un ensemble tout en pierre. En se rapprochant de la deuxième moitié du XIX siècle, les fenêtres semblent s'agrandir en hauteur, les formats de baies deviennent plus carrés et l'on voit apparaitre l'utilisation de " petits bois » pour tenir les vitres : l'image aujourd'hui si caractéristique des « vannes » à petits carreaux est en train de se mettre en place.

Avec 74 ateliers pouvant être rattachés à cette tranche chronologique, la première période représente $6,6 \%$ du corpus. Cette faible proportion s'explique bien évidemment par l'ancienneté des bâtiments, soit disparus, soit profondément transformés. Elle se justifie également par le fait que l'activité coutelière de certains ateliers " primitifs » se déroulait très probablement derrière des fenêtres ordinaires et donc impossibles à distinguer...

\section{Période 2 : de la deuxième moitié du XIXe siècle jusqu'à 1918}

Figure 12

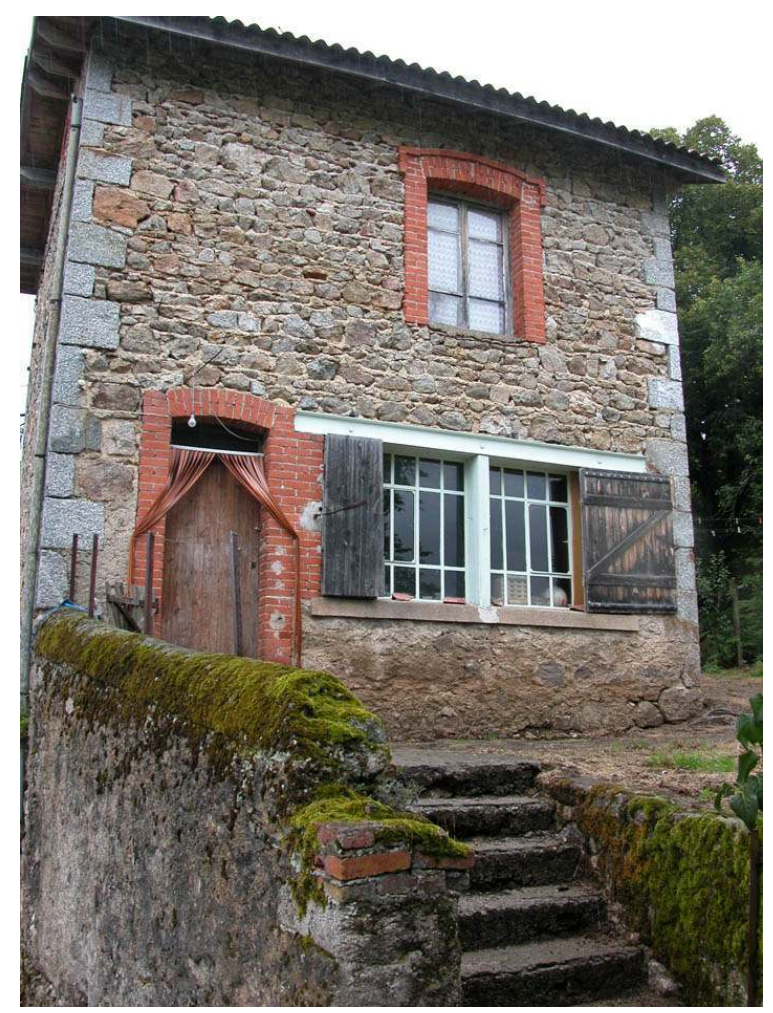

Chapelat, commune de Celles-sur-Durolle. Atelier intégré dans une maison datant de la deuxième période (seconde moitié du XIXe siècle jusqu'à 1918)

Renaud, Bénédicte (c) Inventaire général, Région Auvergne, ADAGP, 2007

Une nette évolution dans la conception des baies d'ateliers se fait à cette époque, au tournant du XIX et du XX ${ }^{e}$ siècle. Si les dernières décennies du XIX ${ }^{e}$ siècle voient encore l'utilisation des matériaux traditionnels de la construction pour toutes les ouvertures (pierre essentiellement, mais le bois n'est pas absent) donnant des élévations encore très homogènes dans de nombreux cas, durant les premières années du $\mathrm{XX}^{\mathrm{e}}$ siècle, de nouveaux matériaux sont largement utilisés, petites briques pleines, linteaux et châssis métalliques, sur des élévations encore en moellons de pierre (enduites ou non) : la différenciation entre maçonnerie et encadrements s'accentue (couleurs plus contrastées, 
lignes plus géométriques) ; les ateliers deviennent ainsi plus repérables depuis l'extérieur des édifices (fig. $\mathbf{n}^{\circ} \mathbf{1 2}$ ).

C'est une période très fortement représentée dans le corpus des repérés; 390 ateliers peuvent être considérés comme ayant été installés durant cette période, soit un pourcentage de $34,7 \%$.

Figure 13

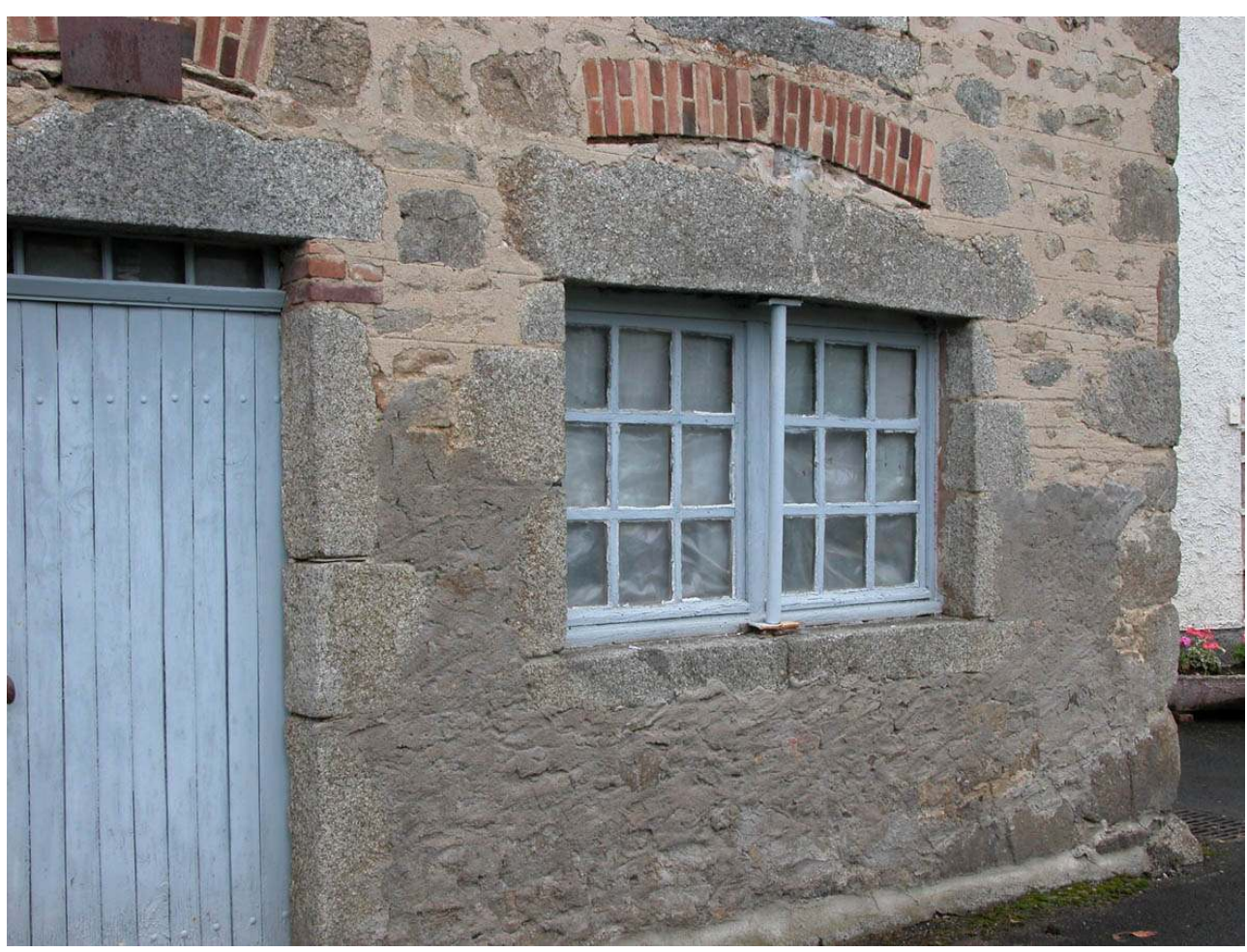

Maubert, commune de Celles-sur-Durolle. Fenêtre d'atelier avec colonnette de renfort destinée à soulager le linteau en pierre déjà fissuré

Ceroni, Brigitte ( I Inventaire général, Région Auvergne, ADAGP, 2007

On a pu noter que nombre de linteaux en pierre d'une grande portée ont été repris à cette époque. Une colonnette "de soutien", souvent métallique, est venue étayer le linteau fissuré, et parfois l'arc de décharge a été consolidé (fig. $\mathbf{n}^{\circ} \mathbf{1 3}$ ).

\section{Période 3 : l'entre-deux-guerres}

Cette période qui recouvre globalement les années 1920, 1930 et le début des années 1940, peut être considérée comme l'âge d'or des ouvriers couteliers à domicile dans la Montagne thiernoise; le pourcentage d'ateliers repérés ne dépasse cependant pas le précédent (il atteint $32 \%$ au lieu des $34,7 \%$ ci-dessus, soit un ensemble de 359 ateliers), mais il est évident que nombre d'ateliers de la période précédente ont continué à être utilisés dans ces années-là, les nouveaux ateliers venant le plus souvent s'ajouter aux précédents et non pas les remplacer. Cela donne donc dans les faits un nombre total assez considérable d'édifices ayant un lien avec la coutellerie et en activité entre les deux guerres mondiales (fig. $\mathbf{n}^{\circ} \mathbf{1 4}$ ). 


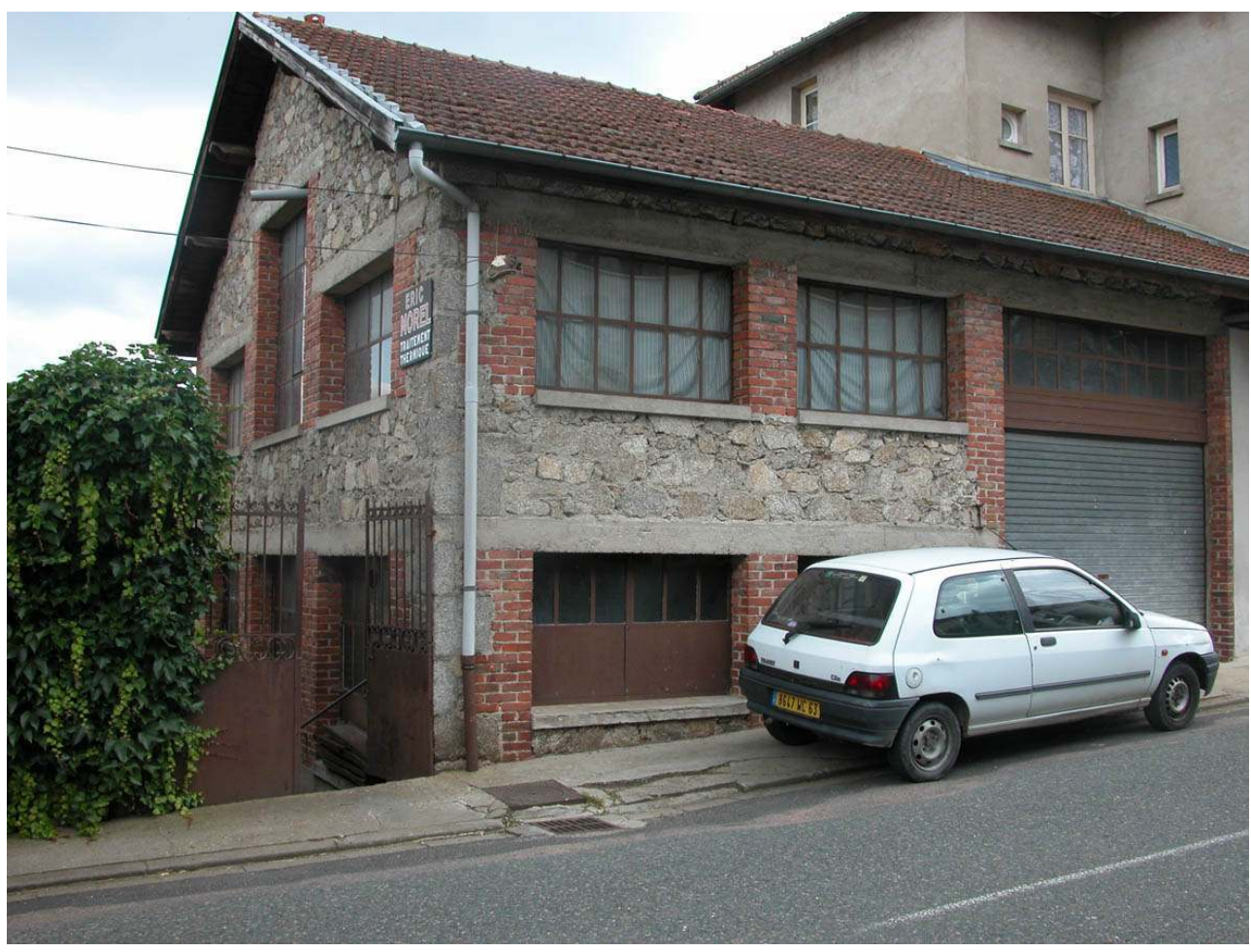

Saint-Rémy-sur-Durolle, bourg. Atelier de la période entre-deux-guerres

Ceroni, Brigitte (c) Inventaire général, Région Auvergne, ADAGP, 2007

53 C'est la grande époque des "vannes » telles que leur définition la plus courante peut les décrire : un châssis métallique plus large que haut, formé par l'assemblage de cornières et composé de petits modules rectangulaires horizontaux en nombre variable, vitrés. Mais le module de base est aussi très souvent employé verticalement, les petites vitres étant alors disposées en hauteur. La fabrication standard des «vannes" métalliques s'est manifestement fortement développée au cours de ces décennies, (un serrurier est encore installé à Celles-sur-Durolle) ; certains ouvriers les ont cependant bricolées eux-mêmes, ce qui est tout compte fait assez naturel pour des artisans travaillant quotidiennement le métal et habitués dans bien des cas à fabriquer leurs outils de travail.

Ces « vannes » métalliques s'intègrent dans des maçonneries encore souvent en moellons de pierre et l'on trouve toujours, dans les années 1920 surtout, des poutres métalliques employées comme linteaux et des piédroits en brique pleine. Cependant l'usage de la brique creuse en piédroits, en chaînages ou même pour toute l'élévation, et celui des linteaux en béton, font leur apparition devenant de plus en plus souvent la norme au fil des années.

\section{Période 4 : de 1945 à la fin des années 1960}




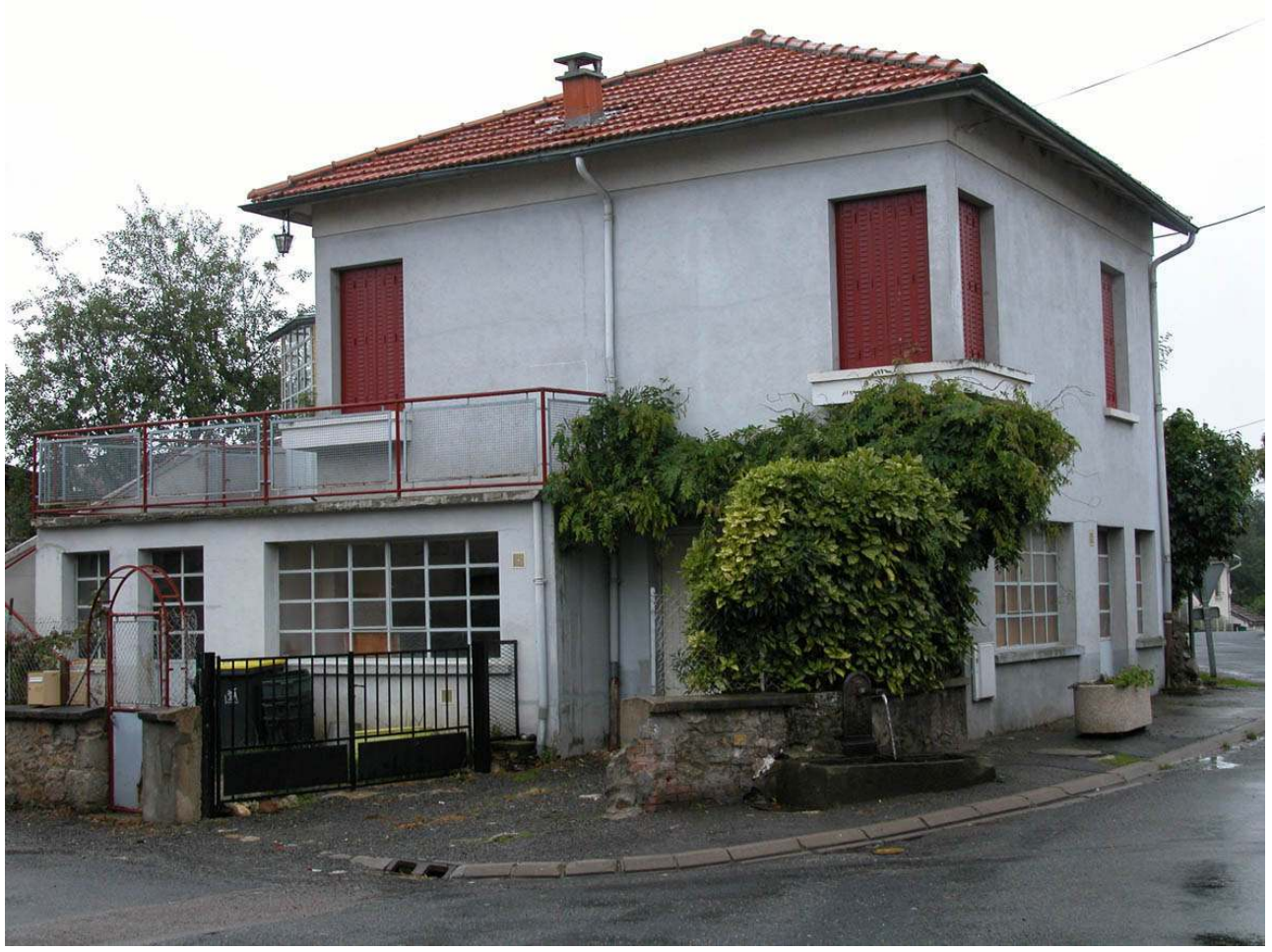

Les Sarraix, commune de Celles-sur-Durolle. Maison avec atelier datant des années 1945-1960 Ceroni, Brigitte (c) Inventaire général, Région Auvergne, ADAGP, 2007

Les années 1950 et 1960 (et peut-être plus spécifiquement les années 1960) représentent une période encore assez prospère pour l'activité des ateliers de coutellerie à domicile : on constate la présence d'un certain nombre de constructions neuves datant de cette époque. 173 ateliers rattachés à cette période ont été décomptés, c'est-à-dire $15,4 \% \mathrm{du}$ corpus total (fig. $\left.\mathbf{n}^{\circ} \mathbf{1 5}\right)$.

Ils se caractérisent essentiellement par l'emploi du béton ou des parpaings de béton, enduits, et leurs murs sont parfois habillés, lorsqu'ils sont installés en rez-de-chaussée d'une villa, d'un parement de pierre en opus incertum - alors à la mode dans la construction - ce qui permet une meilleure intégration au bâtiment. Cette intégration peut aussi passer par la combinaison de deux fonctions, l'une artisanale, l'autre d'agrément, lorsque la dalle couvrant l'atelier accolé à l'habitation fait office de terrasse. Les châssis d'ouverture sont systématiquement en métal, d'un gabarit assez important (ou bien les fenêtres sont multiples) et, bien souvent, les portes sont elles aussi vitrées pour encore plus de lumière.

\section{Période 5 : de 1970 à nos jours}


Figure 16

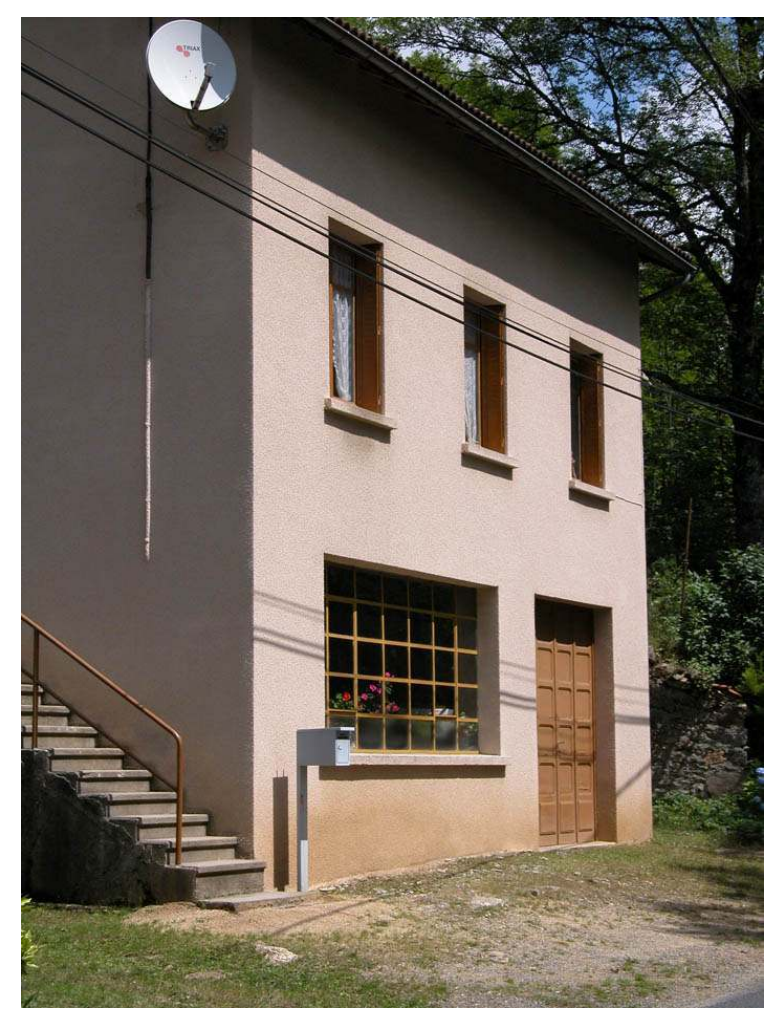

Le Moulin de Riouparet, commune de Saint-Rémy-sur-Durolle. Maison des années 1970 avec atelier Ceroni, Brigitte ( ) Inventaire général, Région Auvergne, ADAGP, 2007

127 éléments ont été retenus, soit l'équivalent de $11,3 \%$ du corpus. D'un point de vue constructif, ces ateliers sont proches de ceux de la période précédente : vitrage important à châssis métallique, encadrements sobres en béton enduit... (fig. $\left.\mathbf{n}^{\circ} \mathbf{1 6}\right)$

Il semblerait que les ateliers construits depuis les années 1970 correspondent plus à des ateliers de fabricants - petits patrons travaillant à leur compte - que d'ouvriers à domicile. Les derniers ouvriers à domicile ont souvent arrêté leur activité dans les années 1990 ou au tout début des années 2000 ; la génération suivante a préféré choisir un autre métier ou rejoindre les usines, regroupées pour la plupart dans les nouvelles zones industrielles (Z.I. de Racine sur la commune de La Monnerie-le-Montel, Z.I. du Mas sur la commune de Palladuc, ...). Les ateliers encore en activité se comptent, par commune, sur les doigts de la main... Rares sont les plus récents: les éléments regroupés ici appartiennent plutôt à la période 1970-1990.

On arrive ainsi à la fin d'une période de développement. Le paysage va se transformer ; les "vannes" ne sont déjà plus un élément "actif» de la Montagne thiernoise mais représentent désormais, sous forme de traces, certes encore nombreuses mais appelées à se raréfier, la mémoire d'un mode d'activité coutelière bien spécifique à ce territoire. 


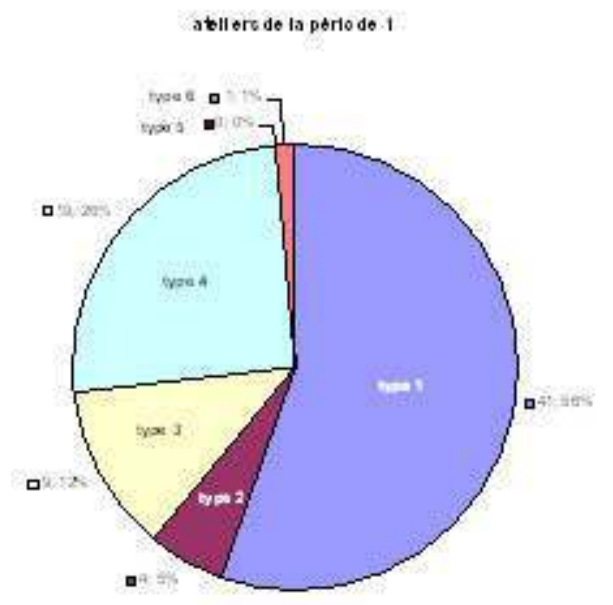

Ateliers de la période 1 (1700-1850) : répartition typologique

Figure 18

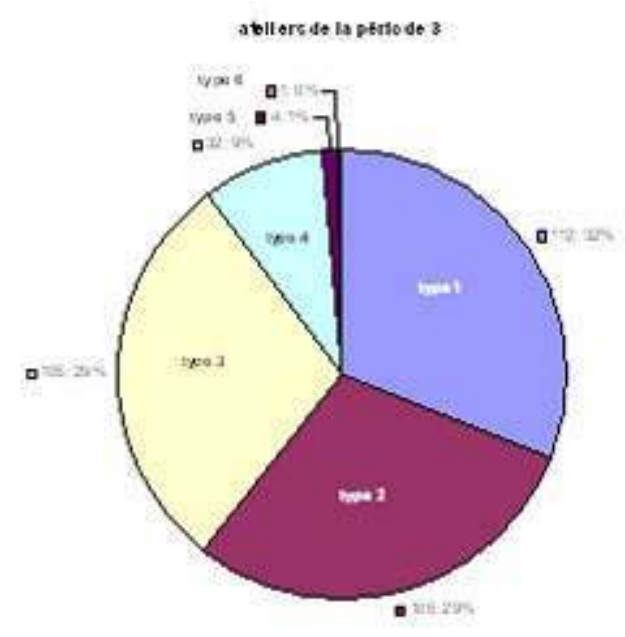

Ateliers de la période 3 (1919-1944) : répartition typologique

Un changement de statut pour les ouvriers?

La comparaison des deux camemberts fait apparaître que les ateliers intégrés au rez-dechaussée de l'habitation (type 1) et les ateliers combinés ou accolés à un bâtiment agricole (type 4) sont bien représentés pendant la période 1 (1700-1850) et sont progressivement «remplacés» par les ateliers de type 2 (indépendant de l'habitation) et 3 (accolé à l'habitation). Il serait tentant d'y voir, malgré les aléas de la conservation qui impose de rester prudent dans nos interprétations, un parallèle entre évolution des formes architecturales et des statuts: d'une activité d'appoint à l'agriculture, le travail du couteau se transformerait en une activité principale (fig. $n^{\circ} 17$, fig. $n^{\circ} 18$ ). 


\section{La répartition géographique des ateliers sur le territoire étudié}

Figure 19

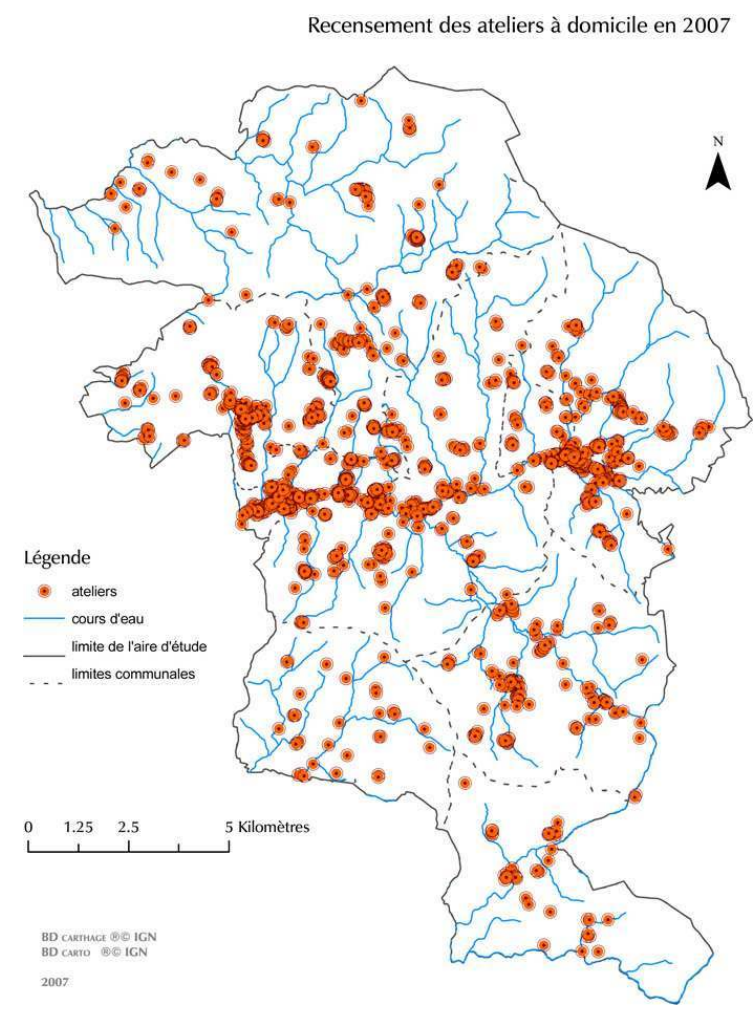

Carte de l'ensemble des ateliers recensés

Fressanges du Bost, Anne (c) Inventaire général, Région Auvergne, ADAGP, 2007

Le système cartographique (SIG) issu de ce recensement permet, outre leur visualisation, de localiser l'ensemble des éléments repérés dans les conditions décrites plus haut et classés selon les modalités qui viennent d'être présentées (fig. $\mathbf{n}^{\circ} \mathbf{1 9}$ ).

Sont ainsi consultables les cartes de répartition de chacun des quatre principaux types d'ateliers à domicile toutes périodes confondues, celles des cinq grandes périodes de construction pour tous types d'ateliers et enfin la combinaison de ces différents critères : ateliers de type 1 construits pendant la période 1 , ateliers de type 1 construits pendant la période 2, etc., pour l'ensemble des périodes et des familles déterminées.

Au vu de cette cartographie, il apparaît en premier lieu que d'une manière générale une grande partie des ateliers les plus représentatifs (types 1 à 4), à l'exception du type 4 (voir ci-dessous), présentent la caractéristique peu étonnante, mais à nuancer selon les époques, d'une concentration le long des cours d'eau - et bien évidemment en premier lieu le long de la vallée de la Durolle ${ }^{26}$, où se trouvent les principaux réseaux routier et ferré-, et dans les gros bourgs (Saint-Rémy, La Monnerie, Chabreloche, Viscomtat...). C'est le cas des ateliers " au rez-de-chaussée ou en soubassement de la maison » (type 1) en particulier de 1920 à 1970 environ, c'est aussi celui des types «isolé ou indépendant " (type 2) ou "accolé à l'habitation " (type 3), aux mêmes époques et encore jusqu'à nos jours. La concentration est particulièrement manifeste quand on isole les ateliers 
construits entre 1945 et 1969. Les répartitions géographiques semblent plus homogènes aux périodes antérieures, en particulier entre 1850 et 1918, c'est-à-dire que la concentration ne devient très nette dans la vallée de la Durolle qu'à partir des années 1910, même si souvent ce sont les communes les plus centrales, au nord et au sud de la vallée de la Durolle qui présentent la plus importante densité de construction, les autres, Saint-Victor-Montvianeix au nord, Sainte-Agathe et Vollore-Montagne au sud, restant un peu à part.

Figure 20

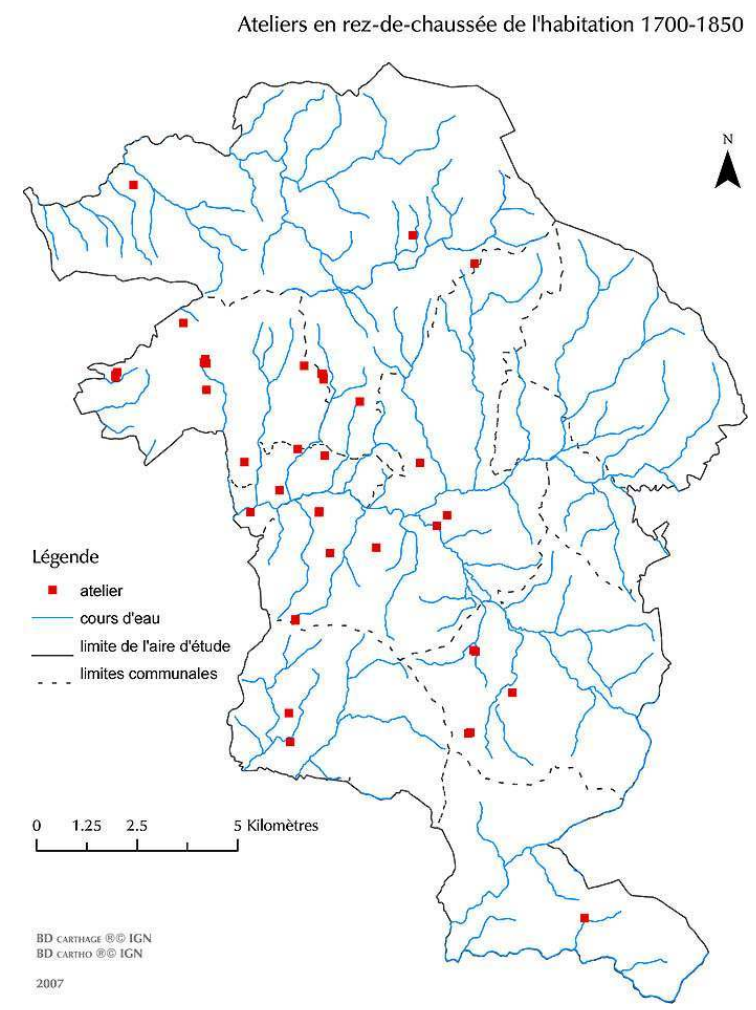

Carte des ateliers en rez-de-chaussée de l'habitation construits entre 1700 et 1850

Fressanges du Bost, Anne ( Inventaire général, Région Auvergne, ADAGP, 2007

Mais il est intéressant de noter que les plus anciens ateliers repérés (période 1 , du XVIII siècle à 1850), en particulier ceux de type 1 (ateliers «au rez-de-chaussée ou en soubassement de la maison ») sont plutôt répartis à l'ouest du territoire étudié, du côté de la commune et de la ville de Thiers, comme si l'influence de Thiers s'était d'abord étendue dans la Montagne en commençant par les zones les plus frontalières, pour gagner ensuite vers l'est (fig. $\mathbf{n}^{\circ} \mathbf{2 0}$ ). 


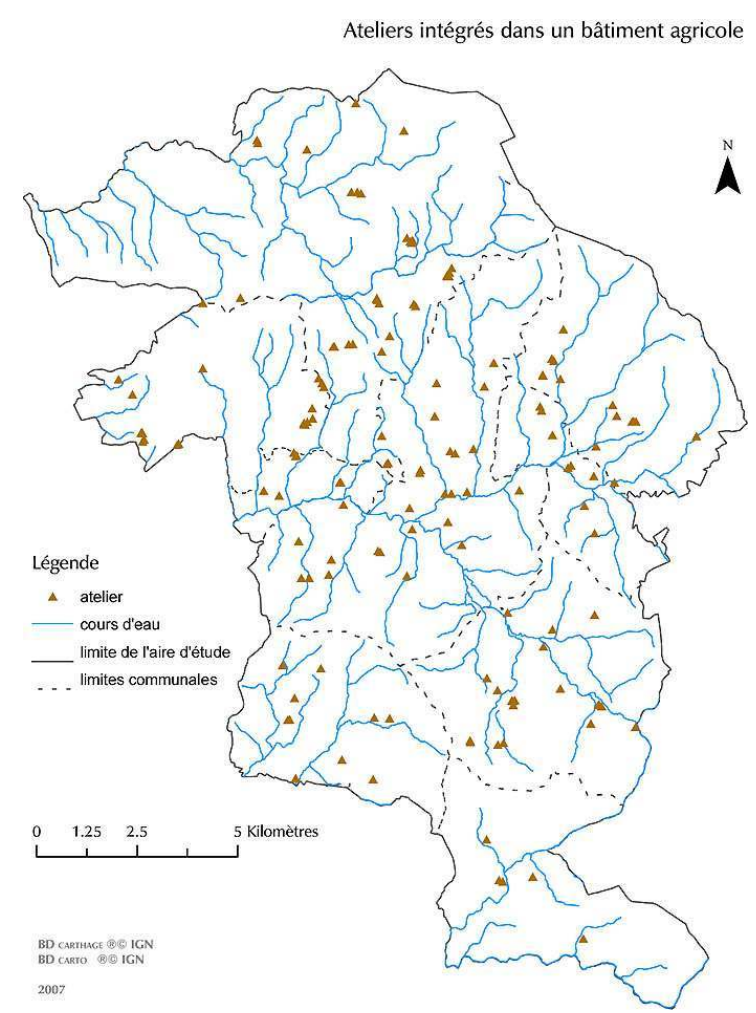

Carte des ateliers intégrés à un bâtiment agricole

Fressanges du Bost, Anne (C) Inventaire général, Région Auvergne, ADAGP, 2007

Autre remarque, les ateliers « combinés ou accolés à un bâtiment agricole » (type 4), ont eux une répartition plus caractéristique en ce qu'elle est totalement indépendante de la rivière de Durolle, et assez homogène sur l'ensemble des communes (sauf à l'ouest de Saint-Victor-Montvianeix) (fig. $\mathbf{n}^{\circ} \mathbf{2 1}$ ). En toute logique, puisque le lien d'implantation se fait seulement avec une activité agricole, il n'existe pas de regroupement particulièrement fort le long de la vallée, contrairement aux autres familles d'ateliers. La période la mieux représentée est celle des années 1850-1918 (période 2): on constate alors la présence du type dans toutes les communes, avec une légère raréfaction aux limites du territoire (nord-ouest, est et sud). Ce type tend ensuite à disparaître dans les périodes suivantes (pendant l'entre-deux-guerres, il n'apparaît plus dans les communes « extrêmes » de Saint-Victor-Montvianeix, Sainte-Agathe et Vollore-Montagne) et devient pratiquement inexistant à partir des années 1950 jusqu'à nos jours, reflétant aussi probablement la raréfaction des exploitations agricoles en activité, ou bien un changement de statut pour les ouvriers, comme nous l'avons vu plus haut ...

Observons encore que les ateliers conservés de la commune de Sainte-Agathe datent presque tous de la période 1850-1918, aucun atelier des périodes postérieures n'ayant été repéré, et tous types confondus (avec une prédilection pour le type $1:$ la fenêtre en rezde-chaussée de l'habitation). Par ailleurs, aucun atelier isolé n'a été repéré à VolloreMontagne alors que les ateliers en rez-de-chaussée ou accolés à l'habitation sont bien représentés.

Au-delà de ces quelques remarques, la confrontation et l'interprétation de ces cartes pourra permettre, nous l'espérons, de multiples observations, qui, liées à d'autres 
phénomènes socio-économiques, géographiques, etc., seront à même de faire progresser la connaissance sur l'histoire industrielle de la Montagne thiernoise (cf. infra De nouvelles interprétations pour une réalité menacée).

\section{Autres bâtiments recensés}

\section{Les rouets}

69 Les rouets ne sont pas à proprement parler des ateliers d'ouvriers à domicile et s'apparentent plutôt à la typologie architecturale des moulins à énergie hydraulique. Ils ont donc fait, dans la mesure du possible, l'objet d'un décompte particulier, qu'ils soient rouets à part entière ou moulins à fonctions mixtes. Ainsi, ce sont 31 bâtiments (ou leurs vestiges), dans ces deux proches catégories, qui ont pu être repérés (fig. $\mathbf{n}^{\circ} \mathbf{2 2}$, fig. $\mathbf{n}^{\circ} \mathbf{2 3}$ ).

Figure 22

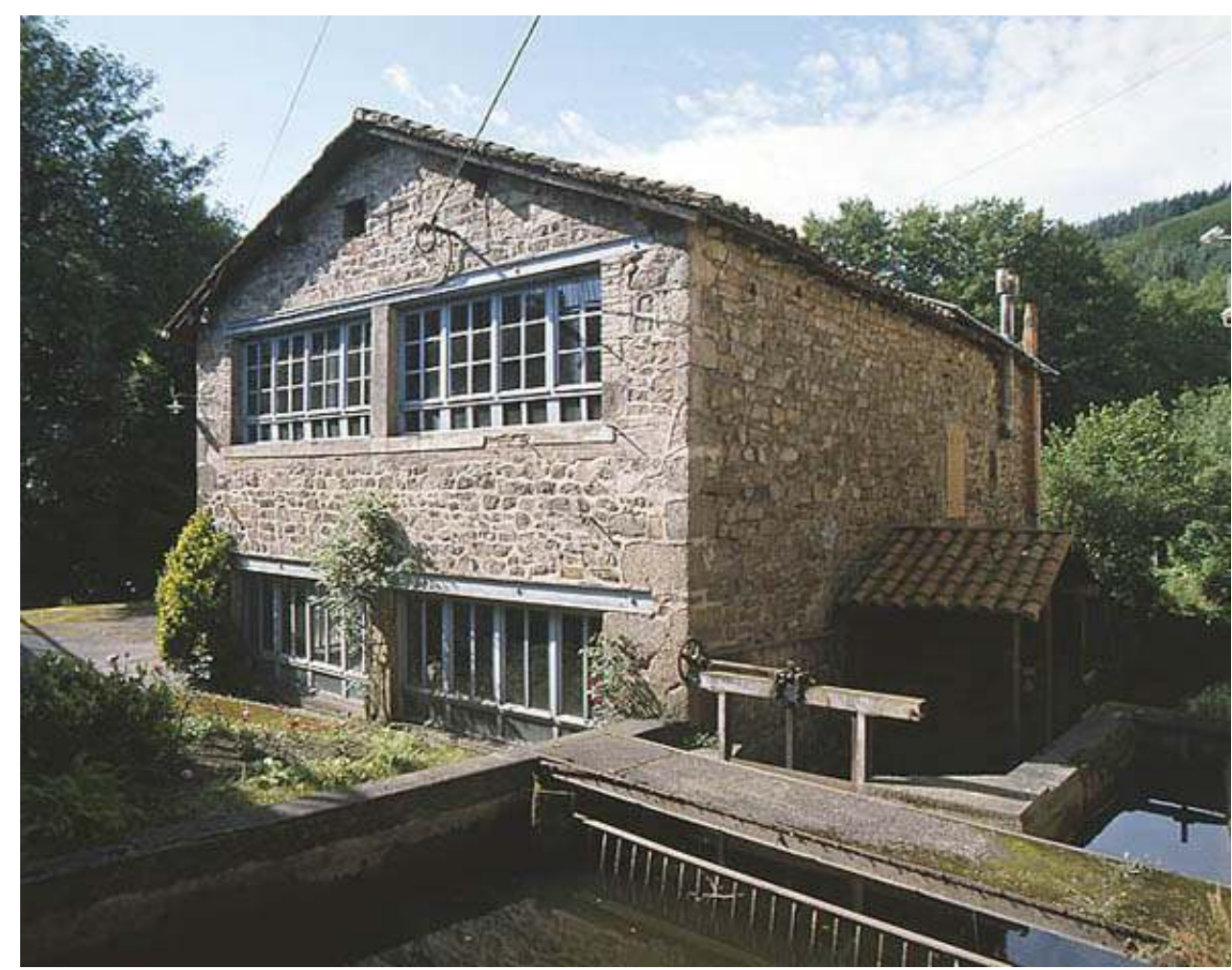

Boulary, commune de La Monnerie-le-Montel. Vue générale du rouet

Périn, Jean-Michel (c) Inventaire général, Région Auvergne, ADAGP, 2007 
Figure 23

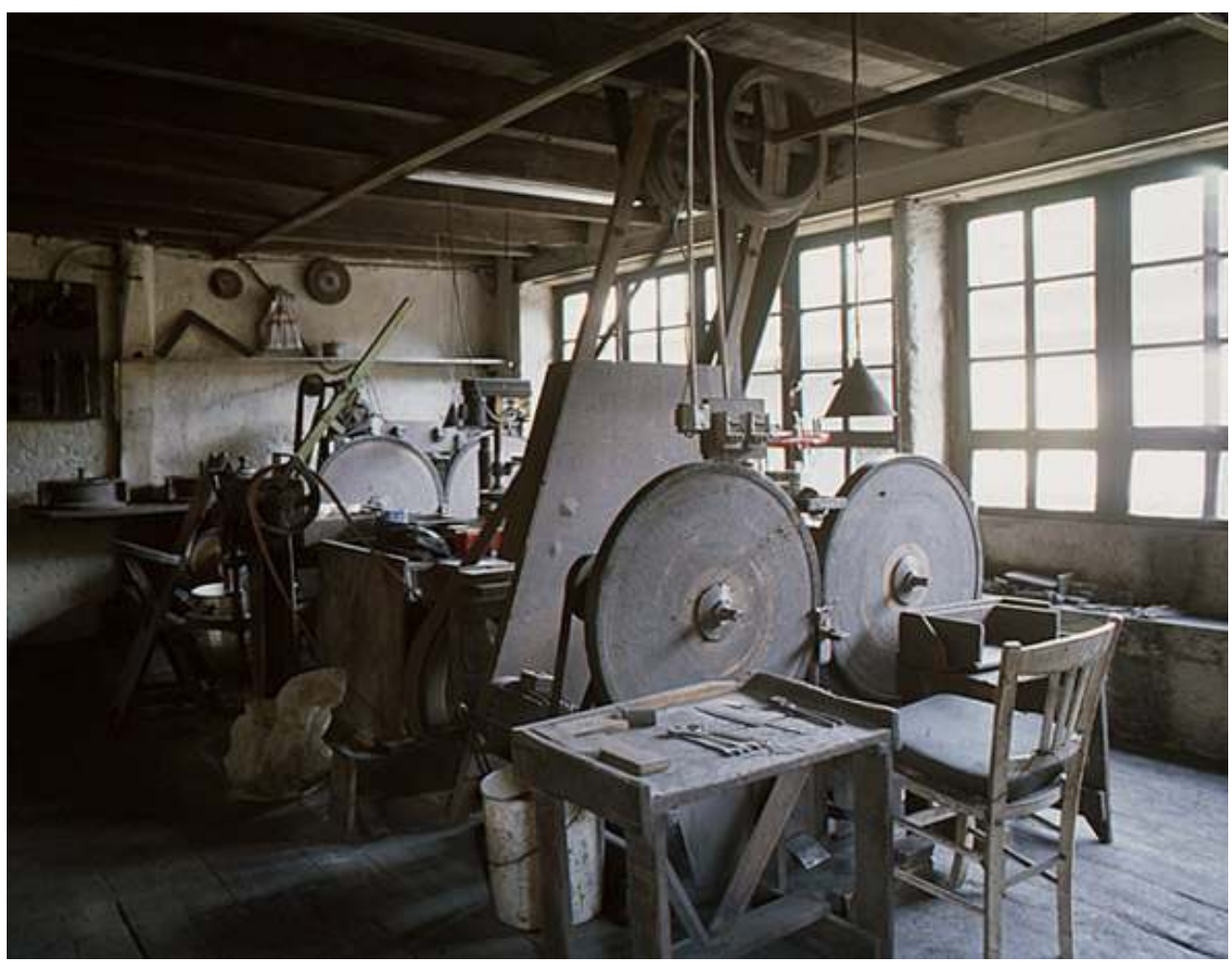

Boulary, commune de La Monnerie-le-Montel. L'atelier de polissage situé au premier étage

Périn, Jean-Michel (c) Inventaire général, Région Auvergne, ADAGP, 2007

Les rouets représentent une catégorie à part entière des lieux voués à la coutellerie. Ce sont des bâtiments totalement consacrés à des fonctions artisanales, le plus souvent ateliers d'émouture en rez-de-chaussée et de polissage à l'étage. Ils fonctionnaient pour beaucoup, du moins à l'origine, grâce à l'énergie hydraulique fournie par des roues verticales installées sur les nombreux ruisseaux et rivières de la région.

71 Au même titre que les moulins à eau, ces édifices sont liés à tout un système hydraulique (retenues d'eau, biefs, chutes, ...) souvent encore décelable sur le terrain.

72 De fait, ce sont des bâtiments généralement isolés en fond de vallée, fréquemment à l'abandon et en ruine aujourd'hui; ils commencent cependant à faire l'objet de restaurations (exemple du rouet de Boulary ${ }^{27}$ ). Leur construction, ou du moins leur installation ${ }^{28}$, remonte en effet souvent au XIX ${ }^{\mathrm{e}}$ siècle, ou au plus tard au début du XIX ${ }^{\mathrm{e}}$ siècle.

D'après des témoignages oraux, trois de ces rouets au moins n'étaient pas destinés à la location de places (rouets de Goutoule et de La Bolloteyre, commune de Viscomtat, rouet du Moulin des Ris, commune de Saint-Rémy).

\section{Les scieries}

La présence de scieries a simplement été notée, à titre indicatif, comme éléments liés à l'histoire de la coutellerie : bois pour les manches de couteaux, pour la fabrication des caisses et boîtes de conditionnement des lames et des couteaux.... ${ }^{29}$ 


\section{Les usines ${ }^{30}$} (lorsque ce n'était pas déjà le cas, puisque les cartes au 1/25000e les distinguent en général). Elles ont été considérées comme un prolongement actuel de l'activité coutelière traditionnelle de la Montagne thiernoise, mais ne s'inscrivant pas précisément dans la problématique de départ. Elles n'ont donc pas fait l'objet d'une photographie systématique.

Elles sont de plus en plus souvent regroupées dans des zones industrielles (Z.I.), comme celle de Racine, par exemple, à proximité de l'autoroute A72 sur la commune de La Monnerie-le-Montel.

\section{De nouvelles interprétations pour une réalité menacée}

Cette opération d'inventaire systématique, en dénombrant les « objets », en établissant une première typo-chronologie rendant ainsi possible les tris selon quelques critères sommaires, permet de mieux penser et appréhender le phénomène. Elle ouvre des pistes pour les futurs chercheurs en autorisant le développement de nos conclusions et la construction de nouvelles hypothèses.

\section{ANNEXES}

\section{Glossaire}

\begin{tabular}{|l|l|}
\hline Terme & Définition(s) \\
\hline Rouet & Usine où l'on émoud et polit les lames de couteaux \\
\hline
\end{tabular}

\section{NOTES}

1. Pour cette étude, consulter: Henry, Anne. Thiers, une exception industrielle. ClermontFerrand : E.P.A., 2004 (coll. Images du patrimoine, $\mathrm{n}^{\circ}$ 229). Pour l'ensemble des notices d'édifices et de machines étudiés, et notamment pour la présentation de l'étude, voir le site: http:// www.culture.gouv.fr/culture/inventai/patrimoine. Voir également, dans la base Mérimée: notice IA63000478. Voir le site qui résume l'étude : Thiers, une exception industrielle.

2. Voir la synthèse de cette étude complémentaire: Potte, Marie-Blanche. Des paysans à l'atelier. Patrimoine coutelier de la Montagne thiernoise. Lyon: Lieux-dits, 2007 (coll. Parcours du patrimoine, $n^{\circ} 326$ ).

3. Archives départementales du Puy-de-Dôme, série M 764. 
4. A titre indicatif encore, on notera qu'à cette même date, 1500 ouvriers seulement sont employés à tailler de la pierre dans la région de Riom et Volvic, période pourtant faste, comme le note le préfet de l'époque, puisque plusieurs chantiers de casernes et celui de la façade de la cathédrale de Clermont battent leur plein. Archives départementales du Puy-de-Dôme, série M 764 .

5. Voir Archives départementales du Puy-de-Dôme, série M 775 et la visite virtuelle : Patrimoine industriel du Jura.

6. Voir Code général des impôts, article 5 , annexe 4 .

7. Des notices d'ateliers à domicile sont néanmoins consultables sur Internet : voir le site http:// www.culture.gouv.fr/culture/inventai/patrimoine; notamment, sur la base Mérimée, les notices : IA63001195, IA63001200, IA63001202, IA63001203, IA63001205 et IA63001211.

8. Voir Archives départementales du Puy-de-Dôme, série P 258. Dépouillement effectué par Maryse Durin-Tercelin, chargée d'études documentaires au service de l'Inventaire.

9. Comme le remarque André David, architecte, missionné par le C.A.U.E. du Puy-de-Dôme, « les fenêtres d'atelier, fréquentes à rez-de-chaussée, ont évité bien des éventrements" (voir "Commune de Celles-sur-Durolle. Diagnostic architectural sommaire », C.A.U.E. du Puy-deDôme, Parc naturel régional du Livradois-Forez, avril 1986). En effet, les châssis de ces baies sont la plupart du temps remplacés mais le besoin de lumière qui se fait ressentir depuis les années Le Corbusier se trouve satisfait par les proportions des « vannes».

10. Lancettes de vétérinaires dont la production est signalée avec insistance dans la Statistique industrielle et manufacturière de l'année 1811. Archives départementales du Puy-de-Dôme, série M 775 .

11. Le montage à domicile, jusqu'en 1965, de machettes à canne à sucre (et de serpettes à raisin) était la spécialité d'un ouvrier du hameau de Mary (commune de Sainte-Agathe).

12. Très logiquement, la plupart des ateliers de menuiserie disposent de châssis en bois plutôt que métalliques... Par ailleurs, la confusion avec les ateliers de menuisiers ne prêtait pas trop à conséquence puisque eux aussi étaient partie prenante dans l'industrie du couteau, en fournissant les manches. Voir, par exemple, la scierie-menuiserie du Becq ou celle de La Fortie (commune de Viscomtat). Par ailleurs, il arrive qu'on ait construit un atelier de polissage de lames et qu'à la génération suivante le même atelier soit agrandi en changeant de destination (menuiserie) : par exemple, à Ricornet (commune de Viscomtat).

13. Encore qu'à Lignières Vieilles (commune de Viscomtat) par exemple, M. Genest s'occupe d'usinage de couteaux dans son atelier de mécanique générale.

14. On pourra observer que les garagistes aussi se fournissent chez les mêmes serruriers mais dans ce cas, la destination du bâtiment est plus clairement annoncée en façade.

15. Les cabanes des commissionnaires ont, elles aussi, contribué à ponctuer l'espace jusqu'à récemment, mais de manière moins parlante.

16. Deux chercheurs (un ingénieur d'études et un conservateur) à temps plein pendant deux mois.

17. Pour 17 écarts (sur 442), des accès indiqués comme privés, ou la présence de chiens de garde agressifs, ont empêché la collecte de données.

18. Ce S.I.G. a été livré aux partenaires de l'opération, accompagné d'un « viewer ». Par la suite, le S.I.G. sera consultable sur le site du service de l'Inventaire général du patrimoine culturel de la région Auvergne.

19. Coste, Georges, de Massary, Xavier. Principes, méthode et conduite de l'inventaire général. Paris : Monum, Éditions du patrimoine, coll. «Documents et méthodes ", $\mathrm{n}^{\circ}$ 9, 2001, p. 41.

20. Un dossier collectif est consultable sur la base Mérimée : voir la notice : IA63001026.

21. Voir Bardisa, Marie, Bonnaud, Pierre, Brunet, Marceline, de Gardelle, Anne-Marie, Leclercq, Jean-Paul. Châteldon entre Dore et Bois noirs. Clermont-Ferrand: association pour le 
développement de l'Inventaire général des monuments et richesses artistiques dans la région Auvergne, 1986, p. 96 (coll. Cahiers de l'Inventaire , $\mathrm{n}^{\circ}$ 6).

22. Voir Breuille, Luc, Dumas, Richard. Maisons paysannes et vie traditionnelle en Auvergne. Nonette : Créer, 1980, p. 397.

23. Voir Bardisa, Marie, Bonnaud, Pierre, Brunet, Marceline, de Gardelle, Anne-Marie, Leclercq, Jean-Paul. Châteldon entre Dore et Bois noirs. Clermont-Ferrand: association pour le développement de l'Inventaire général des monuments et richesses artistiques dans la région Auvergne, 1986, p. 96 (coll. Cahiers de l'Inventaire, n6). Voir aussi Brunet, Marceline, Ceroni, Brigitte, Leclercq, Jean-Paul. Canton de Billom, Puy-de-Dôme. Clermont-Ferrand: EPA, 1991 (coll. Images du patrimoine, $\mathrm{n}^{\circ}$ 95) ; Brunet, Marceline, Ceroni, Brigitte, Leclercq, Jean-Paul. Canton de Maringues, Puy-de-Dôme. Clermont-Ferrand, EPA, 1993 (coll. «Images du patrimoine ", $\mathrm{n}^{\circ}$ 121).

24. Localisation des principaux ateliers datés: Chantelauze (La Monnerie) : 1736, Chabrol (StRémy) : 1821, La Génétie (Viscomtat) : 1833, La Muratte (Palladuc) : 1855, Chailas (La Monnerie) : 1864, Navarron (Ste-Agathe): 1899, Fontbonne (Viscomtat): 1927, Saint-Rémy (bourg): 1936-1940, Pierregrosse (Viscomtat): 1938, La Courtade (Viscomtat): 1946, Chez Bigay (Chabreloche): 1955, Chabreloche (bourg): 1960, Saint-Rémy (bourg): 1964, Chassangues (Celles) : 1965, Chabreloche (bourg) et Ricornet (Viscomtat) : 1970, La Monnerie (R.N.) : 1972, Les Trois Lyndes (Celles) : 1973, Les Hommades (Celles) : 1982. Ces édifices qui portaient une date, qu'il s'agisse d'ateliers ou pas, ont été presque systématiquement photographiés.

25. Communication orale, Paul Chatelain : première époque, du XVI ${ }^{\mathrm{e}}$ siècle à 1914 (distinction nette entre émouleur, monteur et charbonnier,...) ; deuxième époque, 1914 à 1930 (mise en place des usines avec une main d'œuvre qui n'a pas émigré) ; troisième époque, 1930-1980 (évolution vers la plasturgie, l'utilisation de l'inox,...), quatrième époque, 1980-2000 (délocalisation, ...).

26. La proximité de l'énergie hydraulique vaut essentiellement pour les rouets.

27. Voir dans la base Mérimée : notice IA63001150.

28. Ils occupent souvent des bâtiments plus anciens : voir par exemple le cas de Moulin-Planche, commune de Celles-sur-Durolle, où le rouet s'est installé dans un moulin daté 1643 : dans la base Mérimée : notice IA63001151.

29. On pourra néanmoins consulter les notices consacrées aux scieries. Voir dans la base Mérimée : notices : IA63001167, IA63001176, IA63001206, IA63001216 et IA63001217.

30. Les 50 usines (et 13 machines) qui ont été étudiées par Axelle Journaix ont fait l'objet de notices sur les bases Mérimée et Palissy : voir le site http://www.culture.fr/culture/inventai/ patrimoine. Les critères qui ont orienté la sélection sont exposés dans la notice « présentation de l'étude » : voir, sur la base Mérimée : notice : IA63001169.

\section{RÉSUMÉS}

La méthode de l'Inventaire général a été adaptée pour appréhender à l'échelle de dix communes et dans un temps limité la question du travail à domicile des ouvriers couteliers. 1123 ateliers ont été dénombrés depuis l'espace public grâce à la seule présence de leurs fenêtres. La typologie et la datation ont permis en outre de proposer quelques interprétations, voire quelques pistes de recherche, quant à l'activité coutelière qui s'y pratiquait. 
The methods of the French inventory service (Inventaire général du patrimoine culturel) were used as a way of making a rapid survey of ten communes, in order to analyse the question of outwork done by cuttlers. 1,123 workshops were identified, without entering the buildings, simply from the presence of their windows. Typology and dating allowed us to propose some interpretations, even some new avenues for research on cuttlery making activities

\section{INDEX}

Mots-clés : Viscomtat, Vollore-Montagne, rouet, Saint-Rémy-sur-Durolle, Saint-VictorMontvianeix, atelier à domicile, Arconsat, Sainte-Agathe, Celles-sur-Durolle, Chabreloche, Communauté de communes de la Montagne thiernoise, coutellerie, La Monnerie-le-Montel, Montagne thiernoise, ouvrier à domicile, Palladuc, patrimoine coutelier, couteau, lame, manche, tire-bouchon, émouleur, polisseur, industrie à domicile, fenêtre, système d'information géographique

Keywords : grinding wheel, domestic workshop, Montagne thiernoise local authorities, cutlery, Thiernoise mountain, outwork, knife, blade, handle, corkscrew, knife-grinder, polisher, outwork, window, geographical information system

\section{AUTEURS}

\section{BRIGITTE CERONI}

Chercheur. Service chargé de l'inventaire général du patrimoine culturel, Région Auvergne. b.ceroni@cr-auvergne.fr

\section{BÉNÉDICTE RENAUD}

Conservateur du patrimoine. Service chargé de l'inventaire général du patrimoine culturel, Région Auvergne.b.renaud@cr-auvergne.fr 